\title{
Revistas españolas de Documentación en Web of Science: análisis bibliométrico y evolución temática de 2015 a 2019
}

\section{Spanish Information Science journals in Web of Science: bibliometric analysis and thematic evolution from 2015 to 2019}

\author{
Javier Guallar; José-Ricardo López-Robles; Ernest Abadal; Nadia-Karina Gamboa- \\ Rosales; Manuel-Jesús Cobo
}

Cómo citar este artículo:

Guallar, Javier; López-Robles, José-Ricardo; Abadal, Ernest; Gamboa-Rosales, Nadia-Karina; Cobo, ManuelJesús (2020). "Revistas españolas de Documentación en Web of Science: análisis bibliométrico y evolución temática de 2015 a 2019". Profesional de la información, v. 29, n. 6, e290606.

https://doi.org/10.3145/epi.2020.nov.06

Artículo recibido el 23-03-2020

Aceptación definitiva: 14-10-2020
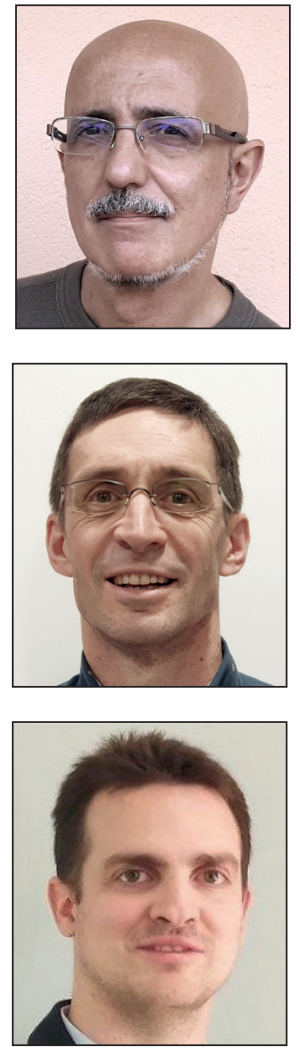

Javier Guallar

https://orcid.org/0000-0002-8601-3990

Universitat de Barcelona. Centre de Recerca en Informació, Comunicació i Cultura Fac. d'Informació i Mitjans Audiovisuals Melcior de Palau, 140. 08014 Barcelona, España jguallar@ub.edu

\section{Ernest Abadal}

https://orcid.org/0000-0002-9151-6437

Universitat de Barcelona. Centre de Recerca en Informació, Comunicació i Cultura Fac. d'Informació i Mitjans Audiovisuals Melcior de Palau, 140. 08014 Barcelona, España abadal@ub.edu

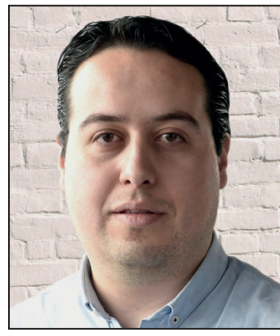
José-Ricardo López-Robles $\triangle$ https://orcid.org/0000-0003-3780-1955

Universidad Autónoma de Zacatecas Unidad Académica de Contaduría y Administración

Jardín Juárez, 147, Centro 98000 Zacatecas, México ricardolopezrobles@outlook.com

\section{Manuel-Jesús Cobo}

https://orcid.org/0000-0001-6575-803X

Universidad de Cádiz

Departamento de Ingeniería Informática

Av. de la Universidad, 10

11519 Puerto Real (Cádiz), España

manueljesus.cobo@uca.es

\section{Resumen}

Las revistas científicas son el instrumento fundamental para la difusión de los resultados de la investigación. Las revistas españolas de Documentación han conseguido una presencia destacada en bases de datos internacionales. A partir del estudio de los artículos publicados en ellas se puede conocer cuál es la evolución temática de la investigación en Documentación, un asunto sobre el que existen pocos estudios. En este artículo se presenta un análisis bibliométrico y temático de las revistas españolas incluidas en el área de conocimiento de Information Science \& Library Science de Web of Science entre 2015 y 2019. Por una parte, se identifica y analiza la producción de las revistas de manera individual y conjunta según los datos disponibles en la Web of Science Core Collection, atendiendo a la productividad de 
los autores, número de citas, organizaciones, países y principales publicaciones. Por otra, se analiza la producción del conjunto de revistas utilizando SciMAT, software bibliométrico de código abierto para la creación de mapas científicos, identificando los temas que han sido objeto de investigación durante el período de análisis, su composición, relación y evolución. Entre los resultados, se aprecia la especialización de las revistas españolas de Documentación en una serie de temáticas que se han agrupado en cinco grandes áreas, por orden de importancia: Redes sociales y medios digitales; Bibliometría y comunicación académica; Open access, open data y big data; Bibliotecas; y Gestión de la información y el conocimiento. Asimismo, estas revistas han ido abriendo el foco temático hacia otras disciplinas, entre las cuales la Comunicación destaca de manera prominente, como queda reflejado en las categorías temáticas establecidas. El estudio permite establecer un marco de referencia para investigadores del área de Información y Documentación, posibilitando la comprensión de nuevas relaciones y oportunidades de investigación, dentro y fuera del área de conocimiento original.

\title{
Palabras clave
}

Revistas científicas; Información y Documentación; ByD; Documentación; Comunicación académica; Bibliometría; Autoría; Citas; Análisis temático; Análisis de co-palabras; Mapa conceptual de evolución; Inteligencia estratégica; Análisis de mapas de la ciencia; SciMAT; Web of Science.

\begin{abstract}
Scientific journals are a fundamental instrument for the dissemination of research results. Spanish Library and Information Science (LIS) journals have achieved a prominent presence in international databases. By studying the articles published in them, it is possible to determine the thematic evolution of research in LIS, a subject on which few studies are available. The current work presents a bibliometric and thematic analysis of Spanish journals included in the Information Science and Library Science category of the Web of Science between 2015 and 2019. On the one hand, the production of the journals is identified and analyzed individually and as a group, according to the data available in the WoS Core Collection, considering the productivity of authors, citations, organizations, countries, and core publications. On the other hand, the production of journals as a whole is analyzed using SciMAT, an open-source software tool developed to perform science mapping analysis in a longitudinal framework by identifying research themes that have been the object of research during the period of analysis as well as their composition, relationship, and evolution. The results highlight the specialization of Spanish LIS journals in a series of topics that can be grouped into five main areas, in order of importance: social networks and digital media, bibliometrics and scholarly communication, open access, open data and big data, libraries, and information and knowledge management. Likewise, these journals have opened up their thematic focus to other disciplines, among which Communication stands out prominently, as reflected in the established thematic categories. This study establishes a reference framework for researchers in the Information Science and Library Science area, making it possible to understand new relationships and research opportunities both inside and outside the original knowledge area.
\end{abstract}

\section{Keywords}

Academic journals; Library and Information Science; LIS; Documentation; Scholarly communication; Bibliometrics; Authorship; Citations; Thematic analysis; Co-word analysis; Keywords analysis; Conceptual evolution map; Science mapping analysis; Strategic intelligence; SciMAT; Web of Science.

\section{Agradecimientos}

Este estudio ha sido realizado en el marco del grupo de investigación consolidado "Cultura i Continguts Digitals" (SGR 2017-422), financiado por la Agència de Gestió d'Ajuts Universitaris i de Recerca (Agaur) de la Generalitat de Catalunya. Los autores agradecen el apoyo del Consejo Nacional de Ciencia y Tecnología (Conacyt, México) y de la Dirección General de Relaciones Internacionales (DGRI, México) para llevar a cabo este estudio. Adicionalmente, este trabajo ha sido apoyado por el Ministerio de Ciencia e Innovación de España bajo la subvención con la referencia PID2019105381GA-I00 (iScience).

\section{Introducción}

Los artículos de revista son el instrumento fundamental para la difusión de los resultados de la investigación en buena parte de las disciplinas científicas (Nederhof, 2006; Sánchez-Gil et al., 2018; Sanz et al., 2007). Los estudios de patrones de citaciones en Documentación (Delgado-López-Cózar, 2002, p. 28) muestran cómo esta disciplina ha ido adoptando el artículo como elemento principal, siguiendo el camino trazado por otras ciencias sociales (Economía, Sociología, etc.) (Sanz et al., 2007). Podemos encontrar una evidencia de ello en los criterios actuales establecidos por la Cneai para superar satisfactoriamente el tramo de investigación (sexenio): se solicita que cuatro de las cinco aportaciones sean artículos de revistas indexadas en los Journal citation reports (JCR) o en SCImago journal rank (SJR) mientras que en épocas anteriores se valoraba especialmente la publicación de monografías (España, 2019). Es por este motivo que actualmente el estudio de los artículos de revista es la vía más completa y detallada para conocer cuáles son las tendencias y la evolución en las líneas de investigación en una disciplina como la Documentación, así como los temas más frecuentes. 
En estos momentos, según un estudio de Abadal (2018), existen 19 revistas científicas activas de Documentación editadas en España, siendo el Boletín de la Anabad (1950) la revista decana. En lo que se refiere a su presencia en bases de datos internacionales, este autor establece tres niveles de reconocimiento: en primer lugar se encuentran Profesional de la información y Revista española de documentación científica [indexadas en Social Sciences Citation Index (SSCI) de WoS (desde 2006 y 2008, respectivamente) y en Scopus]; en segundo lugar Anales de documentación, BiD, Ibersid, Revista general de información y documentación y Scire [indexadas en Scopus y en la base de datos de revistas emergentes de WoS, Emerging Sources Citation Index (ESCI)] y, finalmente, Cuadernos de documentación multimedia, Documentación de las ciencias de la información y Métodos de información [indexadas en ESCI].

Se dispone de numerosos estudios internacionales y españoles que analizan los artículos de revistas de Información y Documentación, ya sea desde el punto de vista de las autorías, los temas o los métodos de investigación. En Guallar et al. (2017) se puede consultar una amplia revisión bibliográfica sobre este tipo de estudios.

En el caso de España, existen diversos trabajos que analizan las autorías y los temas de una o de dos revistas, ya sea la Revista española de documentación científica (Jiménez-Hidalgo, 2007; Pérez-Álvarez-Ossorio, 1997), la Revista general de información y documentación (López-López et al., 2001), BiD (Ollé-Castellà; Porras, 2008), Anales de documentación (González-Alcaide et al., 2008), Profesional de la información (López-Robles et al., 2019a), o de 354 artículos publicados en la Revista española de documentación científica y en Documentación de las ciencias de la información en el período 1977-1994 (Cano, 1999).

Ahora bien, son escasos los estudios más amplios, sobre diversas revistas, y que permiten por tanto una aproximación más precisa de la evolución de la disciplina. En este apartado podemos citar el análisis de los temas de 1.051 artículos del período 2000-2010 (Kawalec, 2013), los estudios de las autorías, temas y métodos de investigación en 580 artículos publicados en las siete revistas españolas indexadas en Scopus en el período 2012-14 (Ferran-Ferrer et al., 2017; Guallar et al., 2017) o el análisis de los temas de 2.247 artículos de autores españoles indexados en revistas de Información y Documentación en WoS en el período 1985-2014 (Olmeda-Gómez; Ovalle-Perandones; Perianes-Rodríguez, 2017). Estos tres últimos trabajos, usando metodologías distintas, nos muestran la evolución temática de la disciplina en los últimos años. Nuestra propuesta se ubica en este ámbito (1.226 artículos de nueve revistas durante cinco años) y pretende no tan solo complementarlo (se basa en las co-palabras y utiliza un software distinto), sino también poner en relación los resultados obtenidos con los ya existentes.

El estudio de los temas tiene diferencias con las investigaciones bibliométricos sobre autorías y productividad. En primer lugar, una mayor complejidad dado que no se basa en el recuento de citas, sino en el análisis de las palabras clave que describen a los artículos y que deben ser normalizadas y agrupadas siguiendo algún criterio temático. En segundo lugar, una mayor disparidad en los procedimientos seguidos para realizar este análisis de las palabras clave y, especialmente, en el instrumento de categorización temática utilizado para agruparlas. Esto explica que los análisis de temas tengan dificultades para poderse comparar entre sí (mientras que los de autorías y productividad son fácilmente comparables) y que además no sean muy abundantes. De todas formas, sus aportaciones son muy interesantes ya que permiten situar con detalle la evolución de una disciplina científica.

A partir de estos antecedentes, el objetivo general de esta investigación es analizar las autorías y los temas de los artículos publicados en las nueve revistas españolas de Documentación que están indexadas en WoS en la categoría Information Science \& Library Science, en los últimos cinco años, el período de 2015 a 2019. Se quiere presentar en primer lugar un estudio bibliométrico descriptivo del contexto para, a continuación, analizar los temas y el mapa de evolución conceptual.

Las preguntas de investigación formuladas son las siguientes:

- ¿Cuál es el panorama general de la investigación en las revistas españolas de Documentación, a partir del análisis de la productividad de autores y de revistas?

- ¿Cuáles son los temas de investigación más destacados en las revistas españolas de Documentación y cuál es el mapa de la evolución conceptual en el período estudiado?

\section{Metodología y preparación de datos}

\subsection{Metodología}

Para realizar este estudio se ha elegido la base de datos Web of Science (WoS). Hay que precisar que WoS ha sido cuestionada por algunos investigadores por su sesgo en pocas publicaciones de idiomas diferentes al inglés, y pocas en disciplinas de Humanidades y Ciencias Sociales (Chavarro et al., 2018; Mongeon; Paul-Hus, 2016). Se pueden encontrar estudios bibliométricos sobre revistas de Documentación que toman como referencia bases de datos como Scopus (Ferran-Ferrer et al., 2017) o LISA (Figuerola; García-Marco; Pinto, 2017), pero también es cierto que son diversas las investigaciones que utilizan WoS para estudios similares al nuestro (Olmeda-Gómez; Ovalle-Perandones; Perianes-Rodríguez, 2017; Sedighi, 2016). Existe por tanto una notable variedad en el uso de fuentes de datos que creemos que contribuye a tener una visión más global y complementaria.

Se ha planteado un enfoque bibliométrico basado en el análisis de los principales indicadores de rendimiento y la confección de su mapa científico, con el fin de identificar los principales componentes, relaciones y su evolución. El análisis 
del rendimiento bibliométrico se centra en el impacto que tienen las publicaciones y los autores según su productividad y citas, mientras que los mapas científicos facilitan la visualización de la estructura intelectual del área de conocimiento a través del uso de las palabras clave incluidas en las publicaciones analizadas. Esto último permite visualizar, entender y describir relaciones difíciles de ver u ocultas entre temas de interés para el desarrollo del campo científico (Cobo, 2012; Cobo et al., 2011; Moral-Muñoz et al., 2020).

En primer lugar se han identificado las revistas españolas indexadas en la categoría Information Science \& Library Science de WoS Core Collection. A continuación se han recopilado y analizado las publicaciones y su información bibliográfica en base a los h-index y H-classics, y por último se ha utilizado el software bibliométrico SciMAT para la creación de mapas científicos. Seguidamente se explica con más detalle.

Por un lado, los datos disponibles en la WoS Core Collection facilitan el análisis de los indicadores de rendimiento más utilizados en la bibliografía: productividad e impacto de autores en términos de citas y publicaciones, organizaciones y países. Esta parte del análisis se ha completado utilizando los índices h-index y H-classics (Alonso et al., 2009; Hirsch, 2005; Martínez et al., 2014; Schreiber, 2007; Schubert, 2007). En primera instancia, se ha utilizado el índice h-index como criterio de referencia para medir productividad e impacto, relacionando el número de publicaciones y las citas de estas. A continuación, se ha utilizado el índice $\mathrm{H}$-classics que hace las veces de criterio imparcial a la hora de sistematizar el procedimiento de búsqueda de citas a través del h-index.

Por otro lado, utilizando el software bibliométrico SciMAT se han identificado los principales temas de investigación de Information Science \& Library Science a manera de mapa científico, que facilita el desarrollo de una metodología que permite:

- Búsqueda e identificación de temas de investigación (Herrera-Viedma et al., 2020; Powell et al., 2016).

- Visualización de los temas de investigación y sus redes temáticas (Callon et al., 1991; He, 1999; López-Robles et al., 2020a).

- Descubrimiento y definición de áreas temáticas (López-Robles et al., 2019c; Sternitzke; Bergmann, 2009).

- Análisis de rendimiento (Cobo et al., 2012).

Los mapas científicos facilitan la visualización de cómo han evolucionado los temas cubiertos por un área de conocimiento, estableciendo los límites de las áreas de investigación en un período de tiempo concreto, lo que permite capturar su estructura conceptual y cognitiva (Cobo, 2012). Previamente a la confección del mapa científico, se caracteriza cada período a manera de red temática y se representa como un conjunto de temas posicionados en un plano divido en cuatro secciones, denominado diagrama estratégico (ver gráfico 1(a)).

Estas redes temáticas se confeccionan según la frecuencia de co-aparición de las palabras clave y se agrupan utilizando el algoritmo de centros simples, uno de los algoritmos más conocidos en la bibliografía. Este algoritmo recupera agrupaciones con etiquetas del nodo más central, es decir, del conjunto de palabras clave con mayor peso que agrupa en sus relaciones aquellas con menor peso. Una vez confeccionada la red bibliométrica de co-palabras, se establece la similitud a partir de la co-ocurrencia de las palabras clave identificadas en el conjunto total de publicaciones, siendo el resultado un conjunto de grupos de palabras clave y sus interconexiones, que se denominan Temas. Por último, estos temas se clasifican en cuatro categorías, según los indicadores de densidad y centralidad de Callon et al. (1991):

- Temas motor (cuadrante superior derecho): desarrollados e importantes para la construcción del campo científico.

- Temas periféricos (cuadrante superior izquierdo): desarrollados internamente, se encuentran aislados del resto de temas, y tienen un papel secundario o marginal en el campo científico.

- Temas emergentes o en declive (cuadrante inferior izquierdo): poco desarrollados, con una tendencia o bien al alza (emergentes) o al retroceso (en declive).

- Temas básicos o transversales (cuadrante inferior derecho): importantes para el campo científico, pero con menor desarrollo.

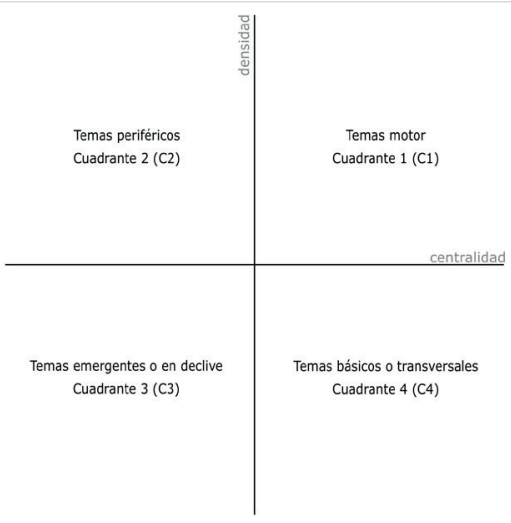

(a)

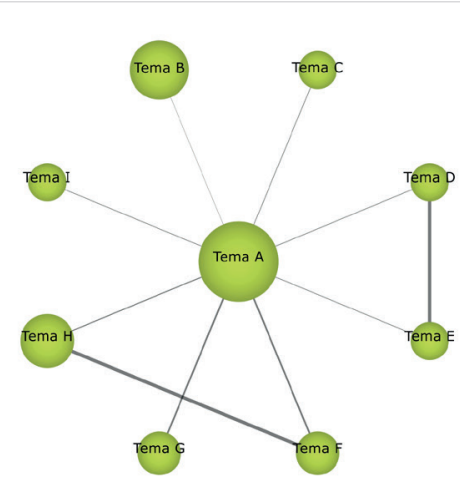

(b)

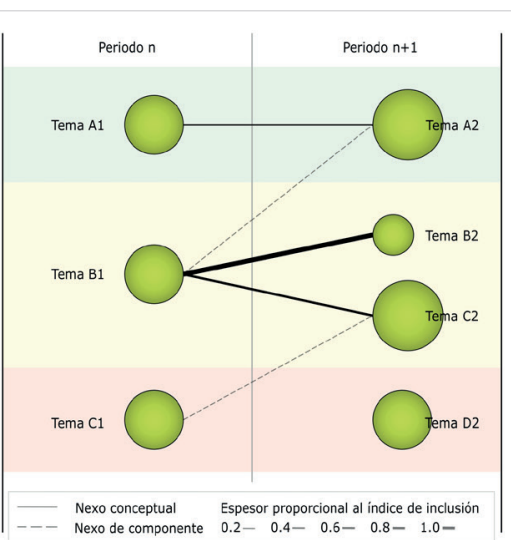

(c)

Gráfico 1. Visualizaciones SciMAT: (a) Diagrama estratégico, (b) diagrama conceptual y (c) mapa científico 
Finalmente, se realiza el mapa científico (ver gráfico 1(c)), que recoge cada diagrama estratégico y relaciona su evolución en el período de tiempo definido. Este mapa se organiza por áreas temáticas para facilitar su análisis y la relación entre los temas de investigación.

\subsection{Preparación de datos}

Después de identificar las revistas indexadas en Information Science \& Library Science de WoS Core Collection se han recopilado las publicaciones y su información bibliográfica utilizando la siguiente consulta avanzada por sus ISSN (impreso y electrónico):

$I S=($ "16992407" OR "13866710" OR “O2100614" OR "19884621" OR "15752437" OR "16977904" OR "19882858"
OR "11321873" OR "11353716" OR “15755886" OR "18880967" OR "1575-9733" OR "2173-1241"),

Refinado por:

AÑOS DE PUBLICACIÓN: (2019 OR 2018 OR 2017 OR 2016 OR 2015) AND CATEGORÍAS DE WEB OF SCIENCE: (INFORMATION SCIENCE LIBRARY SCIENCE) AND TIPOS DE DOCUMENTOS: (ARTICLE OR REVIEW),

Período de tiempo: Todos los años.

Índices:

SCI-EXPANDED, SSCI, A\&HCl, CPCI-S, CPCI-SSH, BKCI-S, BKCl-SSH, ESCI, CCR-EXPANDED, IC 1 .

Esta consulta avanzada recuperó 1.226 publicaciones entre 2015 y 2019, de las cuales 951 (el 77\%) son de acceso abierto. Además, se analizaron las citas de estas publicaciones, siendo la fecha de recogida de datos el 20 de febrero de $2020^{2}$.

Las publicaciones identificadas en la búsqueda avanzada fueron descargadas en formato tipo texto plano y se procesaron utilizando SciMAT, dando lugar a la base de conocimiento que posteriormente fue utilizada para realizar el análisis del mapa científico. De esta manera, la información bibliográfica disponible en WoS (afiliaciones, autores, referencias, fecha de publicación, palabras claves y citas obtenidas, principalmente) está disponible para cada publicación, lo que permite analizar los datos, identificar relaciones y, en consecuencia, obtener mejores resultados en el análisis del mapa científico. Asimismo, para mejorar la calidad de los datos se aplicó un proceso de revisión, en el que se agruparon uno a uno los conceptos que tienen un mismo significado o representan un mismo tema; por ejemplo: "EBOOK", "E-BOOK" y "ELECTRONIC-BOOK" se agruparon como "E-BOOK".

Posteriormente se utilizó el gestor de períodos de SciMAT. La mejor opción a la hora de analizar la evolución de los datos es evaluarlos año a año, aunque en algunas ocasiones es recomendable agrupar estos en períodos para poder generar una masa de publicaciones suficiente para su análisis. En este caso se ha optado por realizar un análisis año a año, por lo que el presente análisis comprende cinco períodos desde 2015 a 2019.

\section{Análisis bibliométrico de rendimiento (autoría y producción)}

En la tabla 1 se presenta la relación de revistas analizadas, incluyendo sus principales indicadores según WoS y el número de identificación con el que se ha realizado la búsqueda avanzada.

Tabla 1. Publicaciones españolas de Information Science \& Library Science en WoS

\begin{tabular}{|c|c|c|c|c|c|}
\hline Revista (ISSN | e-ISSN) & $\begin{array}{c}\text { Publicacio- } \\
\text { nes (acceso } \\
\text { abierto) }\end{array}$ & $\begin{array}{l}2018 \text { Journal } \\
\text { Impact Factor } \\
\text { (2 year impact } \\
\text { factor) }\end{array}$ & $\begin{array}{l}\text { Promedio } \\
\text { de citas por } \\
\text { artículo }\end{array}$ & $\begin{array}{c}\text { Total veces } \\
\text { citado } \\
\text { (sin citas } \\
\text { propias) }\end{array}$ & $\begin{array}{c}\text { Artículos en } \\
\text { que se cita } \\
\text { (sin citas } \\
\text { propias) }\end{array}$ \\
\hline $\begin{array}{l}\text { Profesional de la información (EPI) } \\
(1699-2407 \mid 1386-6710)\end{array}$ & $532(406)$ & $1,505(1,398)$ & 2,70 & $1.437(1.039)$ & $979(775)$ \\
\hline $\begin{array}{l}\text { Revista española de documentación científica (REDC) } \\
(0210-0614 \text { | 1988-4621) }\end{array}$ & $158(158)$ & $0,985(0,949)$ & 1,65 & $260(212)$ & $220(186)$ \\
\hline $\begin{array}{l}\text { Revista general de información y documentación (RGID) } \\
(1988-2858 \mid 1132-1873)\end{array}$ & $134(134)$ & $\mathrm{N} / \mathrm{A}$ & 0,40 & $54(47)$ & $54(47)$ \\
\hline $\begin{array}{l}\text { BiD. Textos universitaris de biblioteconomia i documentació } \\
(1575-5886)\end{array}$ & $103(103)$ & $\mathrm{N} / \mathrm{A}$ & 0,29 & $30(26)$ & $26(23)$ \\
\hline $\begin{array}{l}\text { Ibersid. Revista de sistemas de información y documentación } \\
(1888-0967)\end{array}$ & $79(79)$ & $\mathrm{N} / \mathrm{A}$ & 0,22 & $17(17)$ & $17(17)$ \\
\hline $\begin{array}{l}\text { Scire. Representación y organización del conocimiento } \\
(1135-3716)\end{array}$ & $70(70)$ & $\mathrm{N} / \mathrm{A}$ & 0,33 & $23(18)$ & $23(18)$ \\
\hline $\begin{array}{l}\text { Anales de documentación (AdD) } \\
\text { (1575-2437 | 1697-7904) }\end{array}$ & $57(57)$ & $\mathrm{N} / \mathrm{A}$ & 0,33 & 19 (19) & $19(19)$ \\
\hline $\begin{array}{l}\text { Cuadernos de documentación multimedia (CDM) } \\
(1575-9733)\end{array}$ & $47(47)$ & $\mathrm{N} / \mathrm{A}$ & 0,66 & $31(21)$ & $27(19)$ \\
\hline $\begin{array}{l}\text { Métodos de información (MEI) } \\
(2173-1241)\end{array}$ & $46(46)$ & $\mathrm{N} / \mathrm{A}$ & 0,17 & $8(8)$ & $8(8)$ \\
\hline Total Information Science \& Library Science España & $1.226(951)$ & N/A & 1,53 & $1.880(1.234)$ & $1.296(944)$ \\
\hline
\end{tabular}




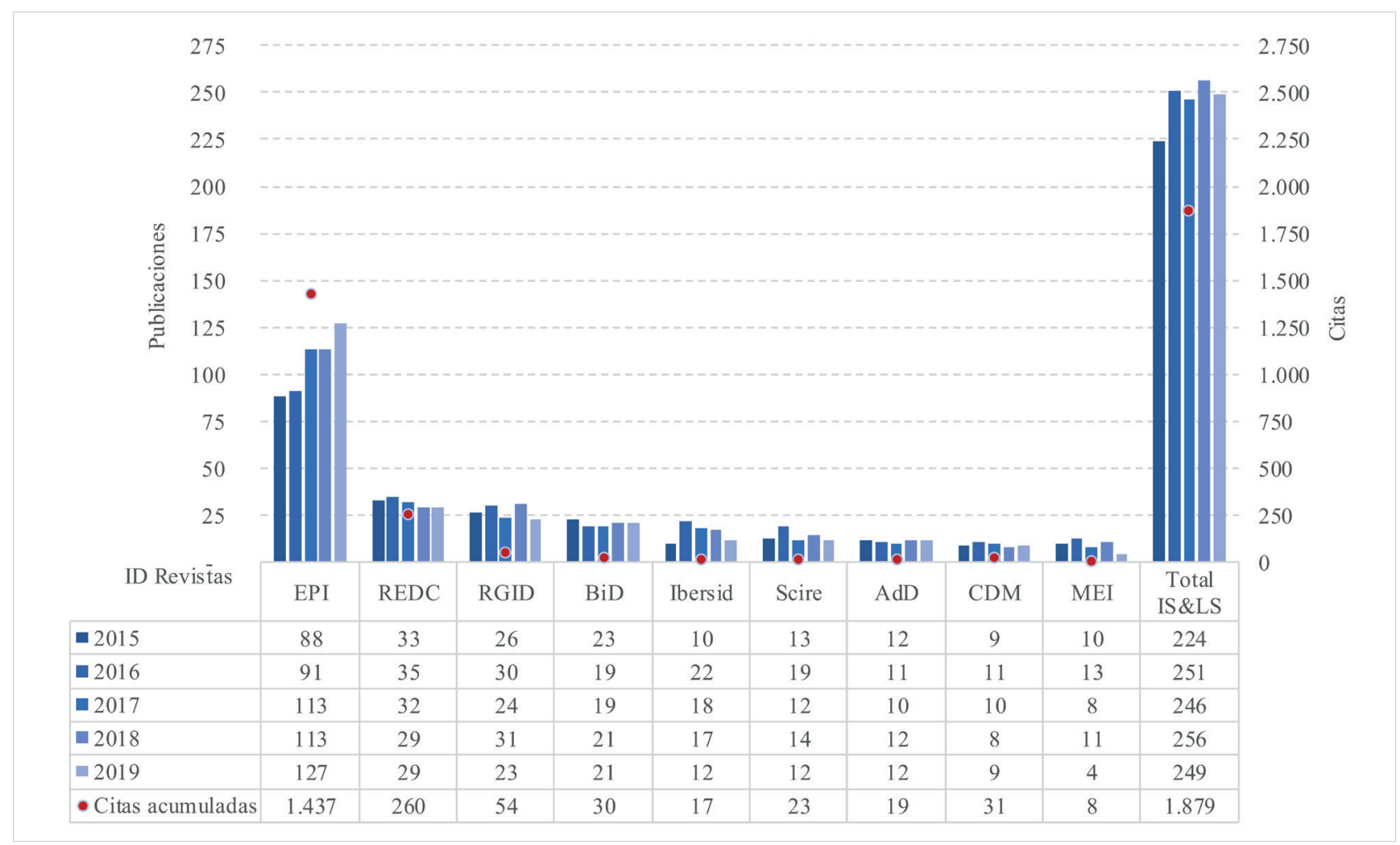

Gráfico 2. Distribución anual de publicaciones y citas 2015-2019 de las revistas españolas de Information Science \& Library Science en WoS

Para la realización del análisis se han considerado los siguientes indicadores: publicaciones, citas recibidas, autores más productivos, publicaciones más citadas, distribución geográfica y organizaciones. El análisis se estructura en tres secciones:

- Publicaciones y citas.

- Autores más productivos y citados, distribución geográfica y organizaciones.

- Publicaciones más citadas.

\subsection{Publicaciones y citas}

En el gráfico 2 se muestra la distribución anual de publicaciones de las revistas analizadas.

En términos de productividad, la revista Profesional de la información se diferencia del resto tanto por tener una producción anual muy superior, entre 88 y 127 artículos, como por ser la única que presenta una tendencia de crecimiento. Las demás revistas tienen producciones anuales entre 10 y 30 artículos aproximadamente y presentan una tendencia de estabilidad o de ligero descenso.

En relación con el impacto, existe una clara diferencia entre las dos revistas indexadas en la base de datos $\mathrm{SSCl}$ con factor de impacto en los JCR y el resto de revistas: Profesional de la información y Revista española de documentación científica superan el umbral de las 250 citas en cinco años, con un total de 1.437 y 260, y un promedio de 2,7 y de 1,65 citas por publicación de media respectivamente, mientras que las otras presentan unas cifras sensiblemente inferiores.

Cabe matizar que las citas recogidas en los dos últimos años muestran una tendencia de mantenimiento o a la baja ficticia; en este sentido, según Wang (2013) las publicaciones más citadas son por lo general las que ocupan años anteriores debido a la ventana de tiempo que existe entre su publicación y en que sean usadas como referencia: se estima que una publicación necesita entre 3 y 7 años para alcanzar su punto máximo de citas.

En suma, es previsible que las dos revistas indexadas en $\mathrm{SSCl}$ mejoren, o al menos mantengan, su rendimiento de productividad y de impacto en relación con su posicionamiento en WoS y que en el resto predomine una tendencia a la estabilidad, con posibilidades tanto de ligeras mejoras como de descensos.
En términos de impacto, existe una clara diferencia entre las dos revistas indexadas en la base de datos $\mathrm{SSCl}$ con factor de impacto en los $J C R$ y el resto de revistas

\subsection{Autores más productivos y citados, distribución geográfica y organizaciones}

Para el análisis bibliométrico es importante identificar quienes son los principales autores en productividad e impacto, así como su distribución geográfica y las organizaciones en las que desempeñan sus investigaciones. 
En la tabla 2 se muestran los autores más productivos y citados para cada revista y para el conjunto en el período 2015 2019, exceptuando los casos en que los autores cuentan con dos o menos publicaciones y menos de 14 citas, siendo el criterio para listar los autores más citados el índice h-index del conjunto, que es 14. Los datos se muestran por orden decreciente, y ante un mismo número de publicaciones, por orden alfabético. Este mismo criterio se aplica a todas las tablas incluidas en la presente investigación. Hay que precisar que, entre las revistas estudiadas, BiD: textos universitaris de biblioteconomia i documentació, es la única que no cuenta con autores con tres o más publicaciones durante el período evaluado, por lo que no aparece en la tabla.

Tabla 2. Autores más productivos y citados por revista y conjunto, 2015-2019

\begin{tabular}{|c|c|c|}
\hline Revista (n) & (N. publicaciones | Porcentaje relativo) Autor(es) & (N. citas | Porcentaje relativo) Autor(es) \\
\hline $\begin{array}{l}\text { EPI }(\text { n. publi- } \\
\text { caciones = } \\
532 \mid \text { n. citas } \\
=1.437)\end{array}$ & $\begin{array}{l}\text { (8 | 1,50\%) Codina } \\
\text { (7 | 1,31\%) Thelwall } \\
\text { (6 | 1,12\%) Arcila-Calderón, Pérez-Montoro, Rodríguez-Bravo } \\
\text { (5 | 0,93\%) Campos-Freire, Casero-Ripollés, Costa-Sánchez, } \\
\text { Goyanes, Guallar, López-García, Manfredi-Sánchez, Martí- } \\
\text { nez-Fernández, Salaverría } \\
\text { (4 | 0,75\%) Alberich-Pascual, Bornmann, Ferran-Ferrer, Gue- } \\
\text { rrero-Solé, Herrera-Viedma, Iglesias-García, Martínez-Pastor, } \\
\text { Míguez-González, Orduña-Malea, Pacios, Rodríguez-Gairín, } \\
\text { Rodríguez-Vázquez, Torres-Salinas } \\
\text { (3 | 0,56\%) Abella, Aguado, Beltrán-Orenes, Busto-Salinas, } \\
\text { Cascón-Katchadourian, Clares-Gavilán, Crespo-Pereira, } \\
\text { De-Moya-Anegón, Delgado-López-Cózar, Eíto-Brun, Freixa, } \\
\text { García-Avilés, García-Orosa, Giménez-Toledo, Giraldo-Luque, } \\
\text { Gómez-Calderón, Halpern, Herrera-Damas, Kousha, Leydes- } \\
\text { dorff, López-Borrull, López-Pérez, Masip, Micó-Sanz, Olve- } \\
\text { ra-Lobo, Ortiz-De-Urbina-Criado, Palau-Sampio, Piñeiro-Otero, } \\
\text { Ramon-Vegas, Repiso, Robinson-García, Rodríguez-Fernández, } \\
\text { Ruiz-Caballero, Ruiz-Rodríguez, Sánchez-Gómez, Suau, Teja- } \\
\text { da-Artigas, Varona-Aramburu, Vianello-Osti, Yubero }\end{array}$ & 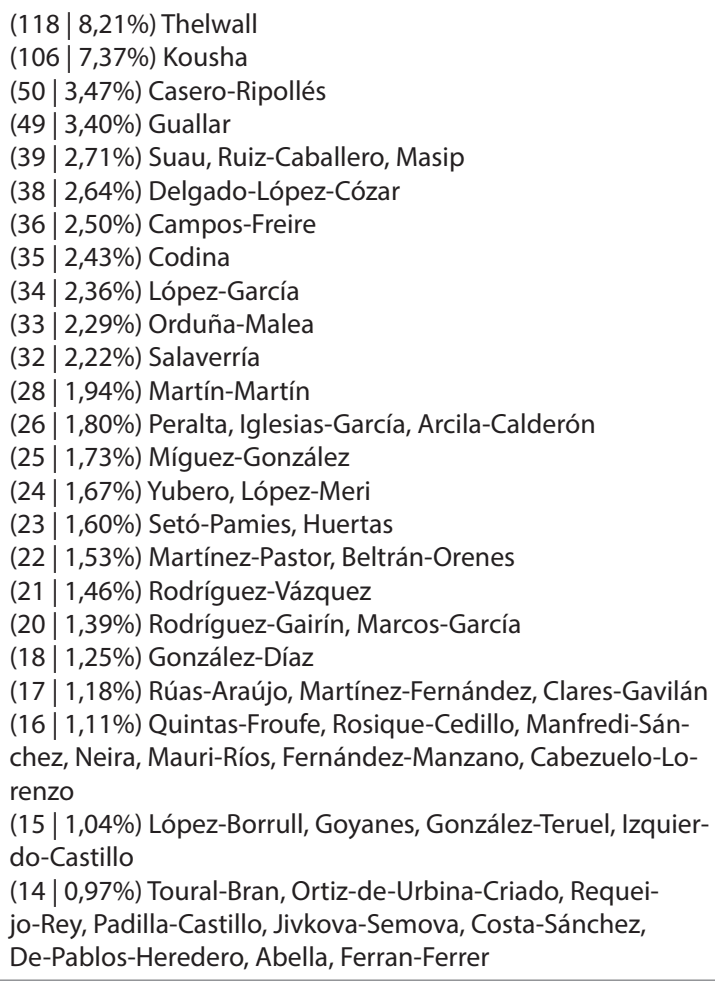 \\
\hline $\begin{array}{l}\text { REDC }(\mathrm{n} . \\
\text { publicacio- } \\
\text { nes }=158 \mid \mathrm{n} \\
\text { citas }=260)\end{array}$ & $\begin{array}{l}\text { (3 1,89\%) García-Zorita, Jiménez-Contreras, Delga- } \\
\text { do-López-Cózar, Luque-Martínez, Marcos-Recio, Martín-Mar- } \\
\text { tín, Montesi, Orduña-Malea, Osca-Lluch, Repiso, Sánchez-Vigil, } \\
\text { Valmaseda-Andia }\end{array}$ & $\begin{array}{l}\text { (39 | 15,00\%) Delgado-López-Cózar, Martín-Martín } \\
\text { (25 | 9,61\%) Orduña-Malea } \\
\text { (15 | 5,76\%) Ruiz-Pérez } \\
\text { (14 5,38\%) Ayllón }\end{array}$ \\
\hline $\begin{array}{l}R G I D \\
(\mathrm{n}=134)\end{array}$ & $\begin{array}{l}\text { (4 | 2,98\%) Moreno, Marcos-Recio } \\
\text { (3 | 2,23\%) Bautista, Olmos }\end{array}$ & \\
\hline $\begin{array}{l}\text { Ibersid } \\
(n=79)\end{array}$ & $\begin{array}{l}\text { (6 | 7,59\%) Marcos-Recio } \\
\text { (5 |6,32\%) Sánchez-Vigil, Olivera-Zaldua } \\
\text { (4 |5,06\%) Lozano, Ortiz } \\
\text { (3 |3,79\%) Cardama, San Juan, Sebastián }\end{array}$ & \\
\hline $\begin{array}{l}\text { Scire } \\
(n=70)\end{array}$ & $\begin{array}{l}\text { (9| } 12,85 \%) \text { Fujita } \\
\text { (5 | 7,14\%) Figuerola, Martínez-Ávila } \\
\text { (4 } 5,71 \%) \text { Berrocal, De Sales, Dos Santos, Guimarães, Moreira } \\
\text { (3 | 4,28\%) Dal'evedove, De Almeida, Tartarotti }\end{array}$ & \\
\hline $\begin{array}{l}A d D \\
(n=57)\end{array}$ & (3 | 5,26\%) González-Fernández-Villavicencio, Sánchez-Vigil & \\
\hline $\begin{array}{l}C D M \\
(n=47)\end{array}$ & $\begin{array}{l}\text { (7 | 14,89\%) Caldera-Serrano, Paz-Enrique } \\
\text { (4 | 8,51\%) Alfonso, Gómez-Crisóstomo, Romo-Fernández } \\
\text { (3 |6,38\%) Arévalo, Faba-Pérez }\end{array}$ & \\
\hline $\begin{array}{l}M E I \\
(n=46)\end{array}$ & (3 | 6,52\%) Paz-Enrique, Puerta & \\
\hline
\end{tabular}




\begin{tabular}{|c|c|c|}
\hline Revista (n) & (N. publicaciones | Porcentaje relativo) Autor(es) & (N. citas | Porcentaje relativo) Autor(es) \\
\hline $\begin{array}{l}\text { Total } \\
\text { Information } \\
\text { Science \& Li- } \\
\text { brary Science } \\
\text { España (n. } \\
\text { publicacio- } \\
\text { nes = } 1.226 \\
\text { |n. citas = } \\
1.879 \text { ) }\end{array}$ & 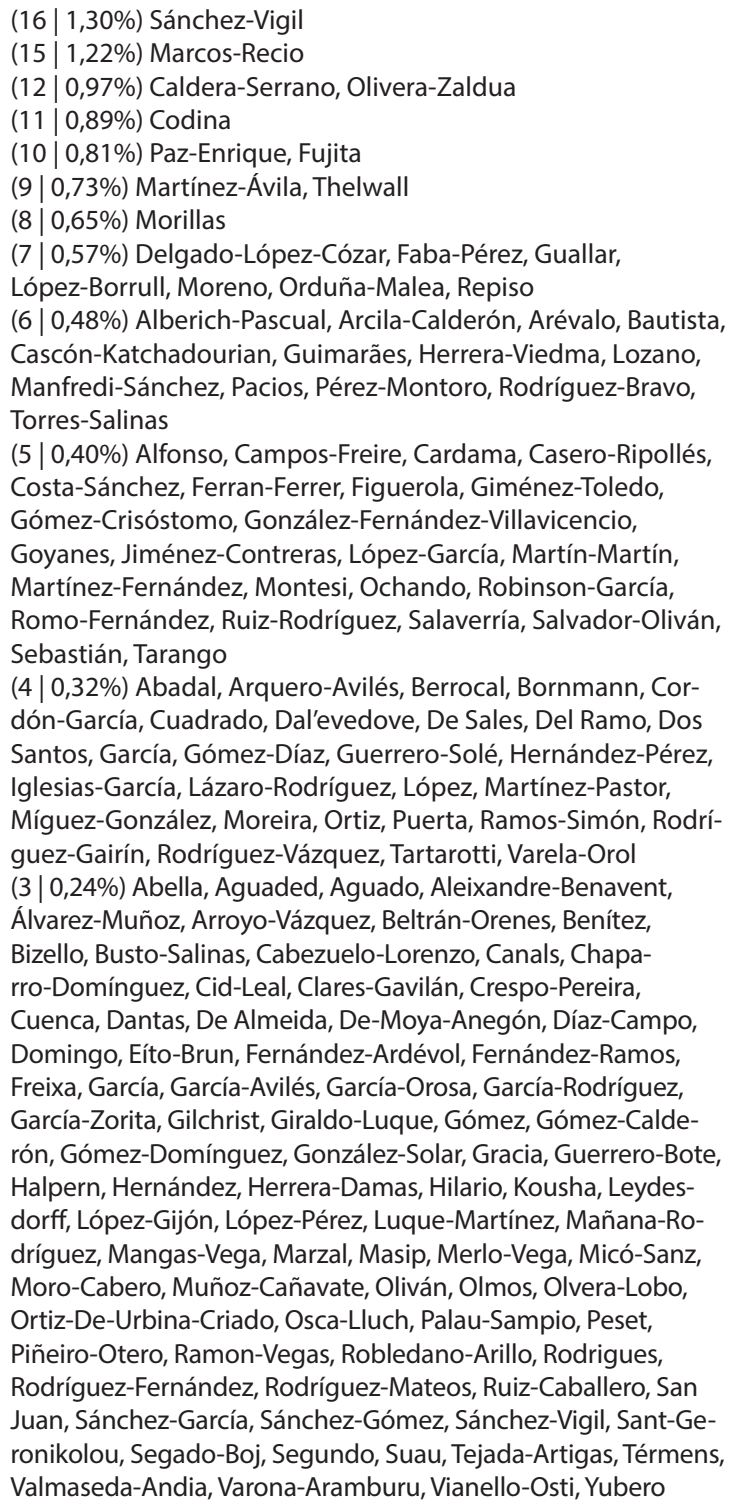 & 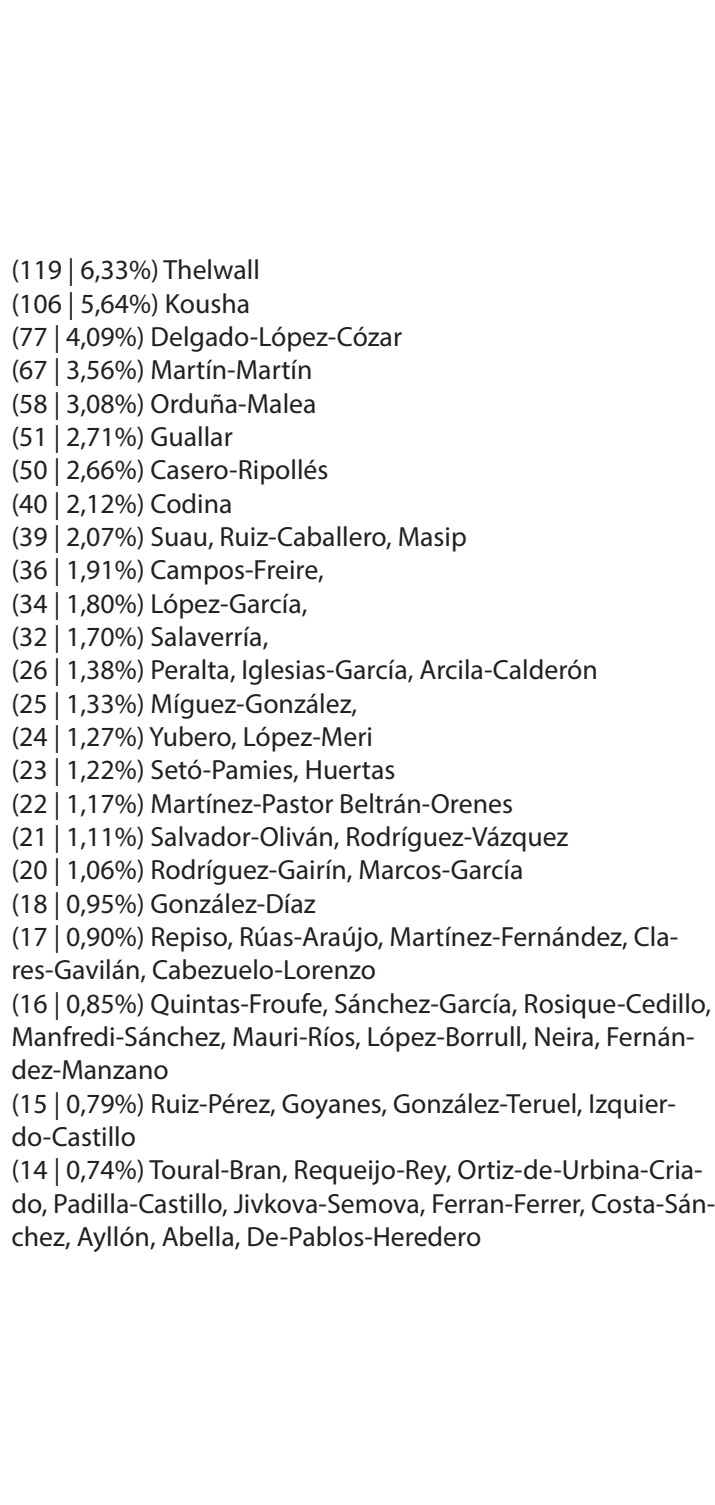 \\
\hline
\end{tabular}

En la tabla 2 se observa en conjunto la importante presencia numérica de autores de la revista Profesional de la información, así como algunas preferencias de determinados investigadores por algunas revistas. Así por ejemplo, si consideramos los cinco investigadores más productivos de Profesional de la información, todos ellos con una productividad entre 6 y 8 artículos (Codina, Thelwall, Arcila-Calderón, Pérez-Montoro, Rodríguez-Bravo) solo coincide uno de ellos (Codina), con la lista de los cinco más productivos en el conjunto de las publicaciones (Sánchez-Vigil, Marcos-Recio, Caldera-Serrano, Olivera-Zaldua y Codina).

Por otra parte, si se comparan los datos de los autores más productivos y más citados de esta tabla 2, destaca en primer lugar la gran diferencia en citación entre los dos grupos de revistas del estudio, observándose que solo entre las dos revistas del SSCI hay investigadores que han alcanzado 14 o más citas. En segundo lugar, varios de los autores más productivos coinciden con los más citados, reflejando con ello una congruencia entre cantidad y calidad (impacto) de las publicaciones de las revistas y del propio campo de conocimiento. Asimismo, entre los investigadores más citados aparecen no solamente varios de los principales autores en producción, sino también otros con menos artículos pero que han obtenido un impacto relevante. Por último, hay que señalar que algunos investigadores que se encuentran en las primeras posiciones entre los más productivos, no están en cambio entre los autores más citados.

En términos de lenguas e internacionalización, las revistas analizadas se publican en cuatro idiomas: español (74,39\%), inglés $(16,48 \%)$, catalán $(5,71 \%)$ y portugués $(3,43 \%)$. La tabla 3 recoge los países y organizaciones más productivos de 2015 a 2019. Es importante mencionar que en las publicaciones en las que los autores tengan adscripción a organizaciones de distintos países, estas computan en los países correspondientes. Las proporciones son similares a las obtenidas en el estudio de Ferran-Ferrer et al. (2017) que también situaba en el 78\% las publicaciones de autores españoles. Las revistas estudiadas cuentan con la participación de 1.897 investigadores de 505 organizaciones y 41 países. España concentra algo más del $78 \%$ de las publicaciones y le siguen Brasil, México, Colombia, Cuba y Reino Unido, con porcentajes de publicaciones cada uno ellos entre el $2 \%$ y $6 \%$ del total. 
Tabla 3. Países, organizaciones españolas y extranjeras más productivas 2015-2019

\begin{tabular}{|l|l|l|}
\hline País (Publicaciones) $\mathbf{n = 1 . 2 2 6}$ & Organización española (Publicaciones) $\mathbf{n = 1 . 2 2 6}$ & Organización extranjera (Publicaciones) $\mathbf{n = 1 . 2 2 6}$ \\
\hline España (963) & Universidad Complutense de Madrid (112) & Universidade Estadual Paulista (34) \\
\hline Brasil (81) & Universidad Carlos III (71) & Universidad Nacional Autónoma de México (20) \\
\hline México (47) & Universidad de Extremadura (61) & Universidade Federal Fluminense (9) \\
\hline Colombia (29) & Universidad de Salamanca (57) & Universidad Central Marta Abreu de Las Villas (8) \\
\hline Cuba (24) & Universidad de Granada (56) & Universidade de São Paulo (8) \\
\hline Reino Unido (20) & Universitat de Barcelona (46) & Universidad de Antioquia (7) \\
\hline Estados Unidos (18) & Universitat Pompeu Fabra (42) & University of Wolverhampton (7) \\
\hline Portugal (17) & Universidad Rey Juan Carlos (42) & Universidade de Coimbra (6) \\
\hline Chile, Ecuador (14) & Universidad de Zaragoza (37) & Universidade Federal de Santa Catarina (6) \\
\hline Argentina (11) & Universidad Politécnica de Valencia (35) & Universidad Autónoma de Chihuahua (5) \\
\hline
\end{tabular}

Las organizaciones más productivas en las revistas analizadas son de origen español, destacando en los primeros lugares las universidades Complutense, Carlos III, Extremadura, Salamanca y Granada. Por su parte, las organizaciones de otros países más destacadas son la Universidade Estadual Paulista en Brasil y la Universidad Nacional Autónoma de México en México.

\subsection{Análisis de publicaciones más citadas}

Para analizar las publicaciones más citadas se plantea un doble enfoque en base al h-index de Hirsch (2005) y al H-classics de Martínez et al. (2014) respectivamente.

Para ello, se utilizó el h-index de 14 obtenido en la consulta realizada en WoS y se identificaron las publicaciones más relevantes, que se presentan en la tabla 4, con indicación de su autoría, tipo de publicación y número de citas en la fecha de recogida de datos.
España concentra algo más del 78\% de las publicaciones y le siguen Brasil, México, Colombia, Cuba y Reino Unido

Tabla 4. H-classics para las revistas españolas de Information Science \& Library Science en WoS, 2015-2019

\begin{tabular}{|c|c|c|c|c|}
\hline Orden & Titulo (autor(es) y año de publicación) & Tipo & Revista & Citas \\
\hline 1 & Web indicators for research evaluation. Part 2: social media metrics (Thelwall; Kousha, 2015b) & Artículo & EPI & 39 \\
\hline 2 & $\begin{array}{l}\text { Web indicators for research evaluation. Part 1: citations and links to academic articles from the web (The- } \\
\text { Iwall; Kousha, 2015a) }\end{array}$ & Artículo & EPI & 36 \\
\hline 3 & $\begin{array}{l}\text { Web indicators for research evaluation. Part 3: books and non-standard outputs (Kousha; Thelwall, } \\
\text { 2015c) }\end{array}$ & Artículo & $E P I$ & 30 \\
\hline 4 & News and social networks: audience behavior (Masip et al., 2015) & Artículo & EPI & 25 \\
\hline 5 & Comunicación de destinos turísticos a través de los medios sociales (Huertas et al., 2015) & Artículo & EPI & 23 \\
\hline 6 & $\begin{array}{l}\text { What do politicians do on Twitter? Functions and communication strategies in the Spanish electoral } \\
\text { campaign of } 2016 \text { (López-Meri et al., 2017) }\end{array}$ & Artículo & $E P I$ & 19 \\
\hline 7 & $\begin{array}{l}\text { Grado de cumplimiento de las Leyes de transparencia, acceso y buen gobierno y de reutilización de los } \\
\text { datos de contratación de la Administración central española (Beltrán-Orenes; Martínez-Pastor, 2016) }\end{array}$ & Artículo & EPI & 19 \\
\hline 8 & $\begin{array}{l}\text { Presencia de las universidades españolas en las redes sociales digitales científicas: caso de los estudios } \\
\text { de comunicación (González-Díaz et al., 2015) }\end{array}$ & Artículo & EPI & 17 \\
\hline 9 & $\begin{array}{l}\text { Producing political content for web 2.0: empowering citizens and vulnerable populations (Casero-Ripo- } \\
\text { Ilés, 2017) }\end{array}$ & Artículo & $E P I$ & 17 \\
\hline 10 & Data management in audiovisual business: Netflix as a case study (Fernández-Manzano et al., 2016) & Artículo & EPI & 16 \\
\hline 11 & $\begin{array}{l}\text { ResearchGate como fuente de evaluación científica: desvelando sus aplicaciones bibliométricas (Ordu- } \\
\text { ña-Malea et al., 2016) }\end{array}$ & Artículo & EPI & 16 \\
\hline 12 & Adaptación de los medios tradicionales a la innovación de los metamedios (Campos-Freire, 2015) & Artículo & $E P I$ & 16 \\
\hline 13 & $\begin{array}{l}\text { El nuevo negocio mediático liderado por Netflix: Estudio del modelo y proyección en el mercado español } \\
\text { (Izquierdo-Castillo, 2015) }\end{array}$ & Artículo & $E P I$ & 15 \\
\hline 14 & $\begin{array}{l}\text { Las revistas universitarias en el marco de los criterios de evaluación de la actividad investigadora en } \\
\text { España (Ruiz-Pérez et al., 2015) }\end{array}$ & Artículo & REDC & 14 \\
\hline
\end{tabular}

En el gráfico 3 se muestra la distribución de las publicaciones más citadas y el impacto generado por estas para el período de 2015 a 2019. 


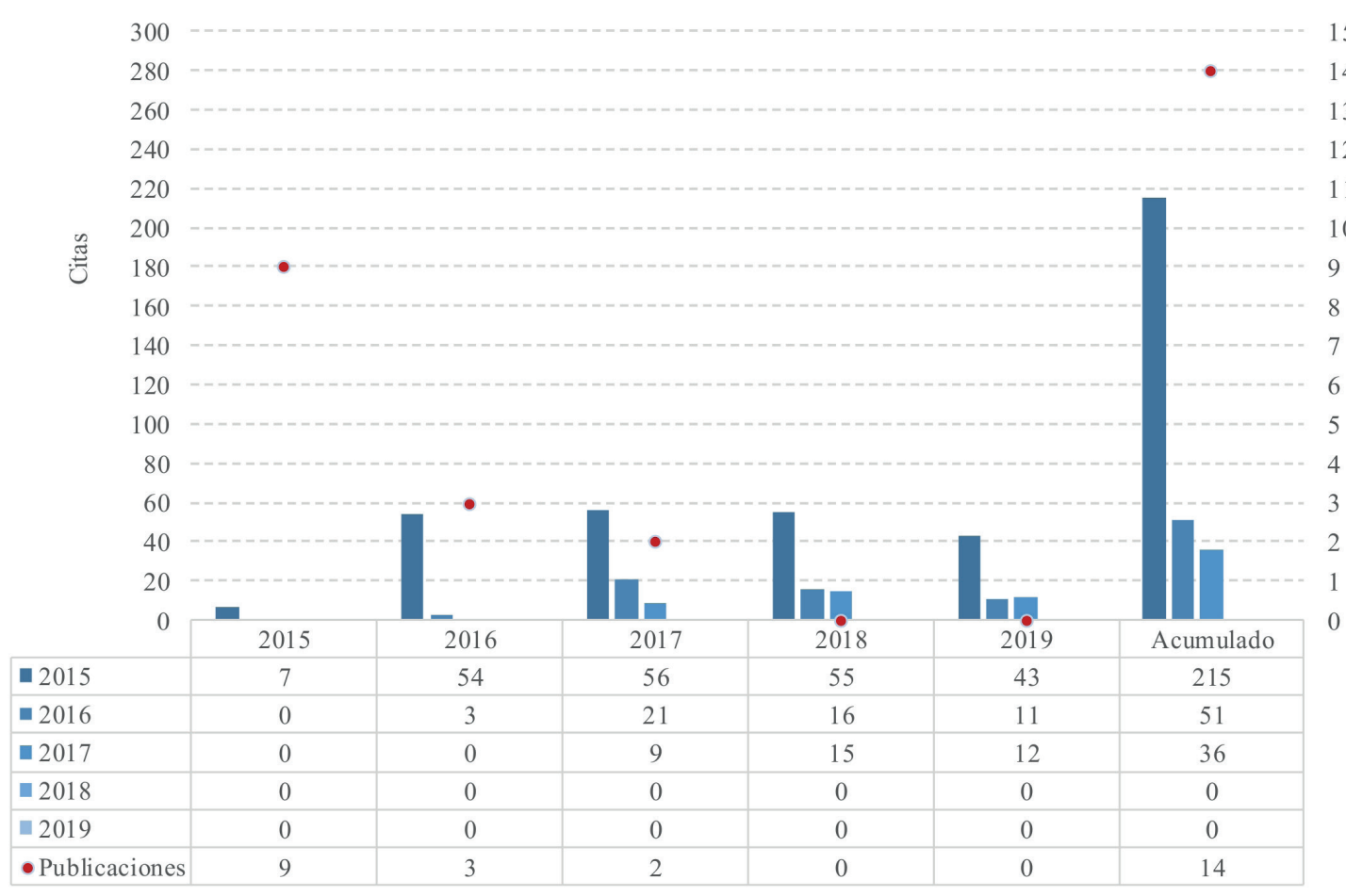

Gráfico 3. Distribución anual de publicaciones más relevantes y sus citas según WoS, 2015-2019

Al igual que en el caso de las publicaciones totales, la distribución de las citas muestra una tendencia positiva y moderada de crecimiento en 2015-2019. Los 14 artículos tienen 302 citas incluidas las autocitas y 299 sin incluir las autocitas, siendo el promedio de citas por artículo de 21,93. Se puede observar que hay artículos que se han mantenido como referentes en un período de 3 y 4 años, y que se prevé mantengan ese comportamiento, lo que sugiere que estas publicaciones forman parte del núcleo de las revistas de Información y Documentación (Wang, 2013). Por último, en cuanto a revistas destaca la presencia abrumadora de Profesional de la información, en todos los artículos menos en uno de la Revista española de documentación científica. Se constata así el diferente comportamiento de los dos grupos de revistas analizados (SSCI o ESCI), acentuándose aún más en este apartado.

Los autores más productivos en las publicaciones incluidas en el H-classics son Kousha y Thelwall con 3 publicaciones (21,42\%), seguidos por Casero-Ripollés, Delgado-López-Cózar y Martín-Martín con 2 publicaciones (14,28\%) y Beltrán-Orenes, Campos-Freire, Clares-Gavilán, Codina, Fernández-Manzano, González-Díaz, Guallar, Huertas, Iglesias-García, Izquierdo-Castillo, López-Meri, Marcos-García, Martínez-Pastor, Masip, Míguez-González, Neira, Orduña-Malea, Peralta, Ruiz-Caballero, Ruiz-Pérez, Setó-Pamies y Suau con una publicación (7,14\%).

España se mantiene como el país con el mayor número de publicaciones entre las más citadas seguida por Reino Unido con $11(78,57 \%)$ y $3(21,42)$ publicaciones respectivamente.

Comparando estos resultados con los mostrados en el apartado 3.2, se aprecia que guardan cierta relación, propiciada principalmente por el desempeño de la revista Profesional de la información.

En cuanto al idioma, los resultados son ahora diferentes que al considerar todo el conjunto de publicaciones, ya que la mitad de estos artículos más citados se han publicado en español y la otra mitad en inglés. Al margen de la constatación de que un número importante de artículos se ha publicado por autores españoles en su lengua, esta distribución indica que las revistas cuentan con la participación de investigadores de otros países, y que, además, algunos autores españoles buscan dar un alcance más internacional a sus investigaciones publicando en inglés. En este sentido, se aprecia un crecimiento que apoya la internacionalización de las revistas.

Por su parte, en lo que a organizaciones con el mayor número de publicaciones más citadas se refiere destacan la University of Wolverhampton de Reino Unido con tres publicaciones y las universidades españolas de Granada, Jaume I y Rey Juan Carlos con dos publicaciones cada una.

\section{Análisis del mapa de evolución}

Tras el análisis de rendimiento de las revistas analizadas, el siguiente paso de este estudio ha sido el análisis de los mapas científicos. Se ha analizado la evolución de dichas revistas identificando y visualizando las principales áreas temáticas, año a año, de 2015 a 2019. Como ya se ha indicado, se ha recurrido al software bibliométrico SciMAT, que puede evaluar el rendimiento de: 
- un área de conocimiento o temática (Alcaide-Muñoz et al., 2017)

- un conjunto de publicaciones (López-Robles et al., 2020b; Moral-Muñoz et al., 2016; Pérez-Cabezas et al., 2018); o

- una revista (López-Robles et al., 2018; López-Robles et al., 2019a).

El primero de estos enfoques es el utilizado en esta investigación, siendo posible el análisis por períodos de más de un año (López-Herrera et al., 2012; Wang et al., 2018) o por años (López-Robles et al., 2018). Esta última variante, apenas presente en la bibliografía, es la que se ha seguido en este artículo.

\subsection{Análisis anual de los principales temas de investigación 2015-2019}

Para visualizar y analizar la evolución temática de las revistas de 2015 a 2019, se han confeccionado los diagramas estratégicos para cada año, así como el mapa de evolución para el área al completo. En el gráfico 4 se muestra el diagrama estratégico para el período 2015-2019. Es importante tener presente que un mismo artículo puede estar incluido en varios temas, en función de las palabras utilizadas para su clasificación.

Tal y como se ha comentado en la metodología, los diagramas estratégicos han permitido clasificar los principales temas de investigación de las revistas analizadas en cuatro categorías, según su relevancia (gráfico 4):

- temas motores (cuadrante C1, superior derecho);

- periféricos (C2, superior izquierdo);

- emergentes o en declive (C3, inferior izquierdo); y

- básicos y transversales (C4, inferior derecho).

Además, cada tema se presenta mediante una esfera en la que el tamaño es proporcional al número de publicaciones, y se incluye entre paréntesis el número de citas que acumulan en el período en cuestión. Es importante mencionar que los temas motores y los básicos y transversales son los que favorecen el desarrollo y la consolidación de un campo de conocimiento por su densidad y centralidad.

Tomando en cuenta los resultados detallados que se muestran en los anexos (tabla A.1), se identifican como temas más dinámicos, con presencia en varios períodos y un rendimiento e impacto considerable los siguientes, por orden: ACADEMIC-LIBRARIES, TWITTER, SOCIAL-NETWORKS, BIBLIOMETRIC-INDICATORS, SOCIAL MEDIA, DIGITAL-MEDIA, BIG-DATA, ELECTORAL CAMPAIGNS, SCIENTIFIC-JOURNALS, PUBLIC LIBRARIES, OPEN-ACCESS, PROFESSIONAL PROFILES, SCIENTIFIC-PRODUCTIVITY, NEWS MEDIA, OPEN-DATA, KNOWLEDGE-MANAGEMENT.

Estos datos permiten visualizar como las temáticas principales en la investigación publicada en revistas de Information Science \& Library Science las siguientes:

- Bibliotecas, destacando entre ellas las universitarias;

- Redes sociales y medios sociales, destacando Twitter;

- Bibliometría e indicadores;

- Medios de comunicación (medios digitales y medios de noticias);

- Big data;

- Campañas electorales (un tema de comunicación política relacionado con su tratamiento en los medios);

- Revistas científicas y producción científica;

- Open access;

- Open data;

- Perfiles profesionales; y

- Gestión de la información y el conocimiento.

A su vez, por su relación entre ellas, los temas anteriores se pueden agrupar en cinco grandes áreas, de las que a continuación se muestra un mapa de evolución y su análisis, y que hemos denominado:

- Redes sociales y medios digitales

- Bibliometría y comunicación académica

- Open access, open data y big data

- Bibliotecas

- Gestión de la información y el conocimiento

\subsection{Mapa de evolución conceptual}

En línea con los diagramas estratégicos, en el gráfico 5 se muestra la evolución de las principales temáticas de las revistas analizadas de 2015 a 2019, agrupadas en las cinco grandes áreas mencionadas. Además, se incluyen los principales indicadores de desempeño para cada una. La calidad de la relación entre ellas se representa por las características de la línea (Sternitzke; Bergmann, 2009). 

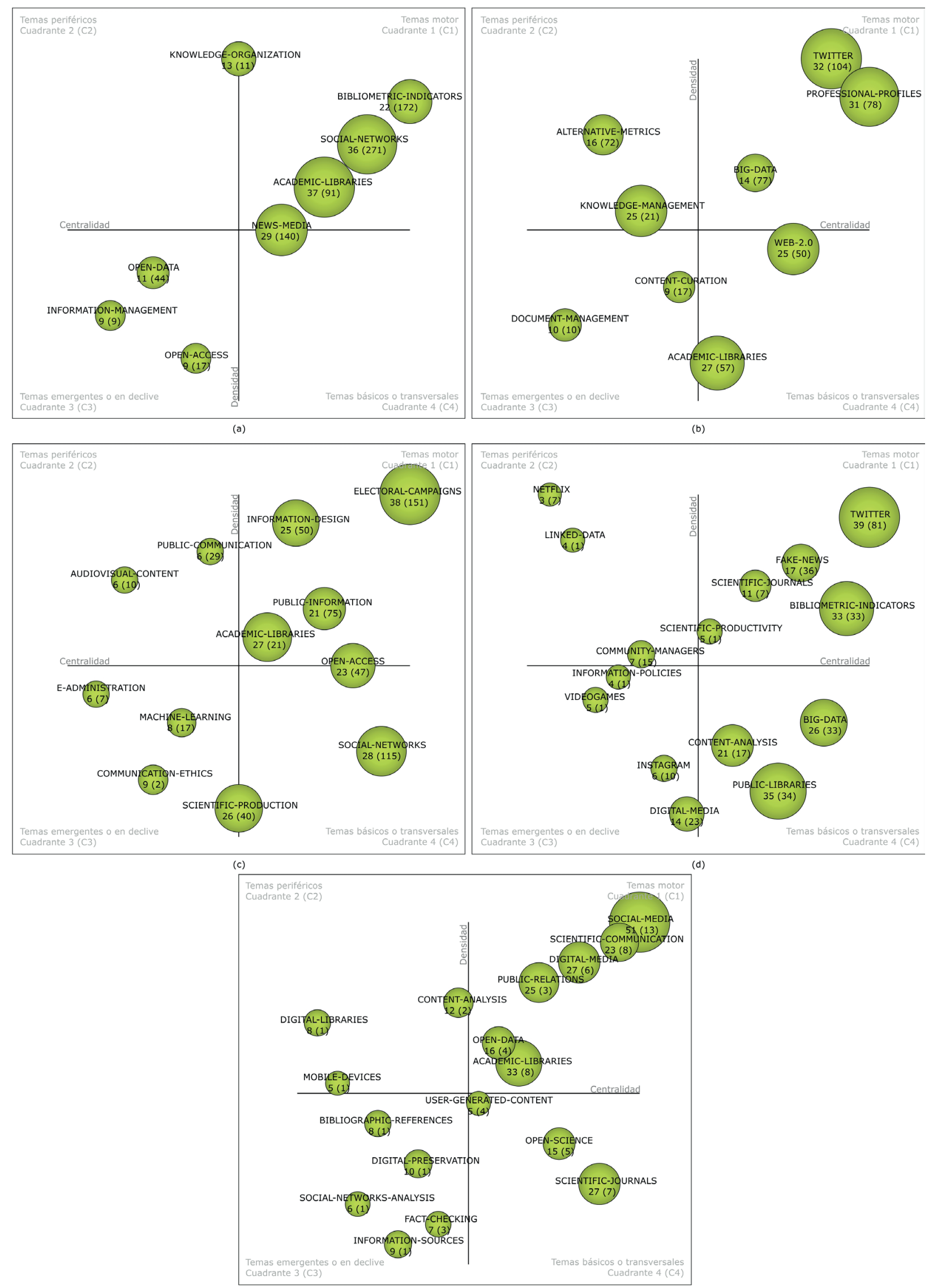

Gráfico 4. Diagramas estratégicos de las revistas españolas de Information Science \& Library Science en WoS Core Collection. (a) 2015, (b) 2016, (c) 2017, (d) 2018 y (e) 2019. 
A nivel general, es importante destacar que el primer y cuarto cuadrantes, relevantes para el desarrollo del campo de conocimiento, concentran el $59,02 \%$ de los temas. Este peso podría considerarse como un reflejo del interés de las comunidades académica y profesional por las áreas temáticas y líneas de investigación desarrolladas en el campo de conocimiento, lo que también se refleja en el crecimiento de la misma y la continuidad que tienen las temáticas incluidas en estos cuadrantes.

A continuación, se analiza cada área (gráfico 5):

\section{Redes sociales y medios digitales (gris)}

Cubre temas motores relacionados con: DIGITAL-MEDIA, ELECTORAL-CAMPAIGNS, NEWS-MEDIA, PROFESSIONAL-PROFILES, PUBLIC-RELATIONS, SOCIAL-MEDIA, SOCIAL-NETWORKS, TWITTER, entre otras. Es la principal área temática del mapa de evolución conceptual. Contiene 422 publicaciones y 1.101 citas.

En términos de estructura y de composición temática, se encuentra repartida en los cuatro cuadrantes durante todos los años analizados (gráfico 4), lo que refleja un equilibrio y una constancia en su desarrollo, aunque presenta una mayor tendencia en temas motor. En suma, es un área que cuenta tanto con una alta producción como una alta citación y que presenta una serie de temáticas que, especializadas o en relación entre sí, abarcan desde las plataformas de medios sociales, con Twitter de manera destacada, a los medios de comunicación digitales, con investigaciones en ámbitos concretos como la comunicación política (elecciones) y las relaciones públicas, además de mostrar una conexión con estudios sobre perfiles profesionales.

Se trata de un área con un enfoque importante en Comunicación, y cuya presencia numérica relevante en el conjunto se puede atribuir en buena medida a las publicaciones de la revista Profesional de la información, situada en WoS también en el área de Communication, algo que estudios anteriores sobre revistas españolas de Documentación ya empezaban a señalar (Kawalec, 2013; Guallar et al., 2017) y que los trabajos recientes sobre esta revista han confirmado (López-Robles et al., 2019a; López-Robles et al., 2019b).

\section{Bibliometría y comunicación académica (azul)}

Sus temas son principalmente: BIBLIOMETRIC-INDICATORS, SCIENTIFIC-COMMUNICATION, SCIENTIFIC-JOURNALS, SCIENTIFIC-PRODUCTIVITY. Es la segunda área temática más representativa, incluyendo 171 publicaciones y 340 citas.

Su estructura y composición temática se comporta de manera similar al área anterior, de forma muy equilibrada y constante. Está delimitada muy claramente, que cubre tanto estudios bibliométricos e indicadores como relacionados con el rendimiento de la actividad científica, con las revistas científicas como principal objeto de estudio, y dentro de la concepción más amplia de lo que se conoce como Comunicación académica.

Estos datos están en línea con los de los estudios realizados en los últimos años sobre artículos de revistas españolas de Documentación (Olmeda-Gómez; Ovalle-Perandones; Perianes-Rodríguez, 2017; Ferran-Ferrer et al., 2017; Guallar et al., 2017), en los que también aparece como un área muy destacada, y marcan una clara continuidad respecto a las observaciones realizadas hace ya 20 años por Delgado-López-Cózar (2000). En esta área ha tenido tradicionalmente y mantiene un papel muy relevante la Revista española de documentación científica, y también se han incorporado otras como Profesional de la información.

\section{Open access, open data y big data (amarillo)}

Sus temas más relevantes son: BIG-DATA, OPEN-ACCESS, OPEN-SCIENCE, OPEN-DATA y PUBLIC-INFORMATION. Situada en tercera posición, con un comportamiento en producción y citación muy similar al del área anterior, agrupando 168 publicaciones y 354 citas.

Su estructura y composición se reparte dentro de los cuatro cuadrantes, especialmente en el tercero. Destacan aquí todas las investigaciones vinculadas con el movimiento Open (access, data y science) siendo entre ellas la línea principal de investigación la de los datos abiertos, con un acercamiento también a los big data, así como a la información pública como el sector más relacionado. Su presencia se puede presumir que está bastante repartida entre las diversas revistas analizadas al tratarse de una temática relativamente reciente que ha interesado a los investigadores de Information Science \& Library Science desde diversos enfoques.

\section{Bibliotecas (rosa)}

Cubre concretamente: ACADEMIC-LIBRARIES, DIGITAL-LIBRARIES y PUBLIC-LIBRARIES. Es la cuarta área temática con 167 publicaciones y 212 citas.

Su estructura y composición se reparte entre el primer, segundo y cuarto cuadrantes, siendo el primero de ellos el más representativo. El enfoque se centra en este caso completamente en las bibliotecas. Esta área es la que se identifica también de manera más directa con la tradicional Biblioteconomía y Documentación: los servicios, las actividades y las estrategias de las bibliotecas, las unidades de información más importantes en la actividad profesional relacionada más estrechamente con el área de conocimiento que se estudia. 


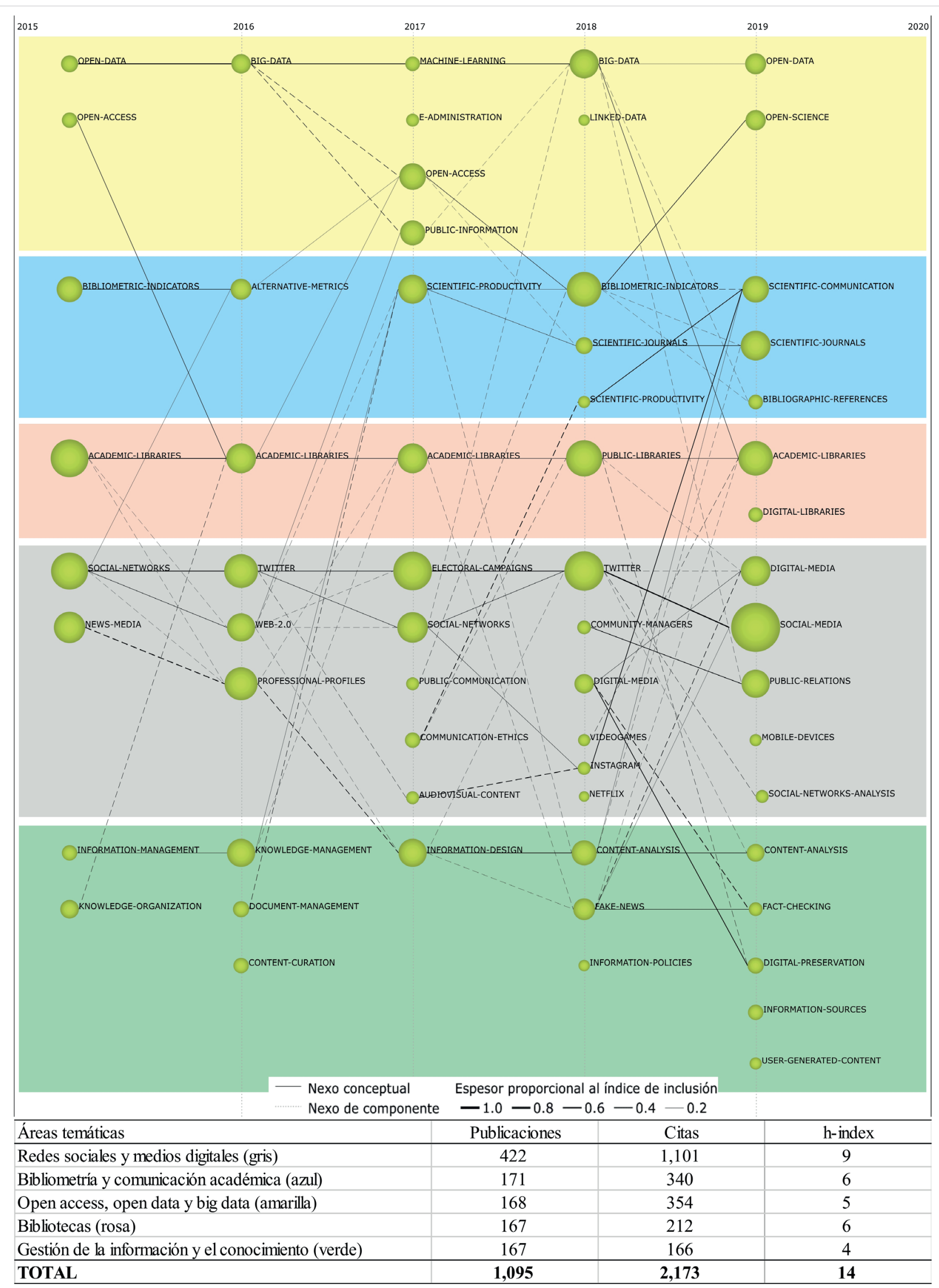

Gráfico 5. Mapa de evolución conceptual de las revistas españolas de Information Science \& Library Science en WoS 


\section{Gestión de la información y el conocimiento (verde)}

Cubre los temas DIGITAL-PRESERVATION, DOCUMENT-MANAGEMENT, INFORMATION-MANAGEMENT, INFORMATION-SOURCES, KNOWLEDGE-MANAGEMENT, KNOWLEDGE-ORGANIZATION, entre otros. Ocupa la quinta posición con 167 publicaciones y 166 citas.

Su estructura y composición se concentra en los cuatro cuadrantes, especialmente en el tercero. Está enfocada en la gestión de la información y el conocimiento y sus implicaciones en las organizaciones. Incluye los conceptos de gestión de la información y de gestión del conocimiento junto a especialidades como la preservación digital y la organización del conocimiento, así como un área temática clásica y muy característica como es fuentes de información. Tiene un comportamiento similar en producción y citación al área anterior de investigación sobre bibliotecas, y vendría a representar la otra cara de la moneda del área de conocimiento que estudiamos, la que tradicionalmente se ha denominado en España Documentación, y ahora también, Gestión de la información.

Estas dos últimas áreas señaladas, sobre bibliotecas y sobre gestión de la información respectivamente, con un nivel de producción similar al de las áreas de bibliometría y open/big data, presentan sin embargo menos citas, debido a que son menos transversales que las anteriores y solo tienen impacto en el núcleo central de la comunidad de ByD, lo cual indicaría un impacto inferior que las anteriores en el desarrollo y la evolución de la investigación del área de conocimiento.

Estos resultados se alinean con los de investigaciones anteriores (Kawalec, 2013; Ferran-Ferrer et al., 2017; Guallar et al., 2017; Olmeda-Gómez; Ovalle-Perandones; Perianes-Rodríguez, 2017; López-Robles et al., 2019a), pero aquí además se muestran algunos matices. Se constata que, si bien existe esa presencia constante en las revistas de Information Science \& Library Science en España de las investigaciones más directamente relacionadas con la profesión de bibliotecarios y documentalistas, estas tienen en cambio un alcance y un impacto más modesto y limitado que las investigaciones bibliométricas y que aquellas más estrechamente vinculadas a áreas emergentes de los últimos años (social media y open access/open data).

\section{En resumen:}

- hay dos categorías temáticas estrechamente relacionadas con el núcleo fundamental (el core) de la Documentación: Bibliotecas y Gestión de la información;

- otras dos categorías son de la capa superior y tienen ya una notable tradición: Bibliometría y Open access/open data;

- por último, Redes sociales y medios digitales es en buena medida una categoría temática importada, que procede de la ampliación hacia el área de Comunicación que han llevado a cabo algunas revistas españolas, en particular Profesional de la información, pero no solamente (también BiD, Cuadernos de documentación multimedia o Scire incorporan artículos de Comunicación).

En relación con este último aspecto, algunos estudios (Ferran-Ferrer et al., 2017) recogen la influencia de otras disciplinas (p.e., Historia y Humanidades en general) en la Documentación, pero en nuestro análisis los descriptores que representan estas temáticas no llegan a aflorar de manera suficiente como para entrar en la categorización que hemos realizado. En estos estudios (Ferran-Ferrer et al., 2017) el peso de la Comunicación era menor (9,3\% del total de artículos) que otras temáticas externas ("Técnicas auxiliares y otras disciplinas", con un 11,5\% del total), aunque el método de análisis era distinto y no se pueden establecer comparaciones fácilmente. Como se ha visto, en nuestro trabajo la presencia de los temas de Comunicación se evidencia de manera más rotunda y creemos que se debe principalmente a la evolución en los últimos años de la revista Profesional de la información hacia una mayor presencia de estos contenidos, como otros estudios recientes también parecen indicar (López-Robles et al., 2019a; López-Robles et al., $2019 b)$.

\section{Conclusiones}

Actualmente existen nueve revistas españolas indexadas en la categoría Library Science \& Information Science de WoS, estando dos de ellas en SSCl y las otras siete en ESCI. Se trata de una proporción destacada de títulos si lo comparamos con otras disciplinas de ciencias sociales, tal y como se ponía de manifiesto en un estudio global sobre la presencia de revistas científicas españolas en bases de datos internacionales (Somoza et al., 2017).

En productividad destaca la revista Profesional de la información que publicó el $43 \%$ de los artículos indexados en el período 2015-2019. En lo que se refiere a citas recibidas vuelve a destacar esta revista junto con la Revista española de documentación científica (ambas en SCCI) porque reciben del 76\% y del 14\%, respectivamente. La evolución de las publicaciones españolas de Library Science \& Information Science en WoS de 2015 a 2019 en conjunto es positiva, aunque dicha evolución viene marcada principalmente por estas dos revistas presentes en SSCl. Además, dado el aumento de publicaciones y citas recibidas, así como los temas de investigación identificados y su evolución en WoS, es posible esperar que el uso e interés de la comunidad científica en este campo de conocimiento se mantenga o siga creciendo durante los próximos años.

En el análisis bibliométrico de rendimiento cabe destacar que las revistas analizadas cuentan con la participación de 1.897 autores de 505 organizaciones y 41 países entre 2015 y 2019. Los autores más productivos son Caldera-Serrano, Marcos-Recio, Sánchez-Vigil, Codina, Paz-Enrique, Fujita, Martínez-Ávila, Thelwall, Olivera-Zaldua y Morillas, mientras que los más citados son Thelwall, Kousha, Martín-Martín, Orduña-Malea, Delgado-López-Cózar, Guallar, Casero-Ripollés, 
Codina, Masip, Suau y Ruiz-Caballero. Existe una coincidencia por tanto parcial entre los autores más productivos y los más citados. Si por una parte, esto refleja un cierto equilibrio y la calidad de las publicaciones incluidas en este campo científico de conocimiento, por otra parte se constata el impacto muy superior de las dos revistas incluidas en $\mathrm{SSCl}$ respecto a las de $\mathrm{ESCl}$. Esto se aprecia en que todos los investigadores más citados publican en estas dos revistas, Profesional de la información y Revista española de documentación científica y en que algunos de los más productivos, al no publicar en ellas, no se encuentran entre los más citados.

Las organizaciones más productivas en España han sido las universidades Complutense, Carlos III, Extremadura, Salamanca, Granada, Barcelona, Pompeu Fabra, Rey Juan Carlos, Zaragoza y Politècnica de València, mientras que a nivel internacional destacan la Universidade Estadual Paulista (Brasil) y la Universidad Nacional Autónoma de México (México).

Las revistas españolas de Información y Documentación presentan un h-index de 14, indicador que refleja el número de publicaciones referentes por su impacto en el desarrollo del campo de conocimiento en cuestión. Este grupo de 14 publicaciones tienen 302 citas (incluidas las autocitas) presentando una media de 21,93 citas cada una y se concentran todas en Profesional de la información, menos una de la Revista española de documentación científica. Los autores con más de una publicación entre las más citadas son Kousha, Thelwall, Casero-Ripollés, Delgado-López-Cózar y Martín-Martín. En cuanto a la internacionalidad es destacable que de los cinco primeros autores, dos no son españoles. Entre las organizaciones con mayor productividad entre las publicaciones más citadas destacan la University of Wolverhampton con tres publicaciones (algo relacionado con los autores antes mencionados) y las de Granada, Jaume I y Rey Juan Carlos con 2 publicaciones cada una. Lo anterior coincide con los resultados obtenidos inicialmente, lo que refleja una coherencia entre la productividad y el impacto de los autores y organizaciones.

Por otro lado, el análisis temático revela la especialización en una serie de temas que se han agrupado en cinco grandes áreas, por orden de importancia: Redes sociales y medios digitales; Bibliometría y comunicación académica; Open Access, open data y big data, Bibliotecas; y Gestión de la información y el conocimiento.

De ellas, la primera, Redes sociales y medios digitales, es claramente dominante en producción y en citación, lo que se puede relacionar, además de por el empuje evidente de la investigación en estas temáticas, con la alta productividad y citación de la revista Profesional de la información, que también publica trabajos de Comunicación, enfoque mayoritario de los trabajos de esta área.

Las áreas de Bibliometría y comunicación académica y de Open access, open data y big data tienen comportamientos similares, mostrando ambas un notable dinamismo en citación. Se trata en el primer caso de un área claramente consolidada de la investigación de Library Science \& Information Science en España, que cuenta además con una publicación altamente especializada como es la Revista española de documentación científica, así como con el interés de buena parte del resto de revistas; y en el segundo caso, de uno de los campos de estudio que más ha crecido en los últimos años, en relación directa con la expansión del movimiento Open: acceso abierto, datos abiertos, ciencia abierta, etc.

Las dos últimas áreas, Bibliotecas y Gestión de la información y el conocimiento, también mantienen un comportamiento similar, presentando ambas un nivel de citación inferior al resto. Agrupan las investigaciones más directamente vinculadas a lo que se pueden considerar los dos grandes sectores profesionales del área de conocimiento: las bibliotecas y la documentación, y aunque mantienen un perfil constante en la producción, tienen una incidencia menor que el resto en el impacto de la investigación del área de conocimiento.

En conjunto se aprecia un cierto equilibrio en la investigación en todas las áreas temáticas observadas, ya que no existe ninguna de ellas en situación de claro retroceso o declive, ni tampoco se observan unas enormes diferencias entre ellas. Mantienen así un perfil bajo pero estable las dos áreas más relacionadas con la profesión de biblioteconomía y documentación, se sitúan en el nivel intermedio de producción y de citación un ámbito tradicional de la investigación en ByD como es la bibliometría y otro enfocado a temáticas de claro empuje en los últimos años como es el movimiento open; y por último, destacan en el nivel más alto de producción y de citación temáticas vinculadas con la comunicación como son las redes sociales y los medios digitales.

Se ha visto por tanto, en lo que se refiere a este último aspecto, la importante relación que existe entre las disciplinas de la Documentación y la Comunicación en las revistas de ByD españolas. Esta relación se puede deber por un lado a la apertura de las principales revistas a recibir investigaciones que pueden formar parte de ambas áreas de conocimiento, pero también a la posición que juegan las ciencias de la información en el desarrollo del conocimiento actualmente. 
Finalmente, de cara a investigaciones futuras, se puede señalar que este artículo es de los primeros en la bibliografía especializada en presentar el uso de SciMAT para estudiar la evolución de un campo de conocimiento en un país, facilitando la comprensión de cómo interactúa un grupo de revistas en un entorno similar. En este sentido, se plantea realizar en el futuro más análisis de manera individual para entender cuál es la aportación de cada revista y sus oportunidades, conociendo las tendencias que existen en el campo general. Finalmente, esta investigación permite, a los equipos de trabajo de las revistas y a los propios investigadores, conocer mejor el campo científico de cara a orientar futuras investigaciones, establecer colaboraciones y potenciar con ello el desarrollo científico y académico.

\section{Notas}

1. Atendiendo a que la búsqueda avanzada se realiza a través de los ISSN de las revistas, en la Tabla 1 se recogen los títulos completos de las mismas.

2. Una revisión manual permitió detectar algunos errores en la indexación de Web of Science, como la ausencia de 5 artículos de la revista Cuadernos de documentación multimedia de 2019, que deberían estar en la base de datos. No obstante, al no llevar a cabo una revisión sistemática de todos los posibles errores de indexación de WoS, se decidió mantener el estudio con los registros disponibles en la WoS.

\section{Referencias}

Abadal, Ernest (2018). “¿Cómo han cambiado BiD y las revistas españolas de documentación en los últimos veinte años?". BiD: textos universitaris de biblioteconomia i documentació, n. 40.

https://doi.org/10.1344/BiD2018.40.11

Alcaide-Muñoz, Laura; Rodríguez-Bolívar, Manuel-Pedro; Cobo, Manuel-Jesús; Herrera-Viedma, Enrique (2017). "Analysing the scientific evolution of e-Government using a science mapping approach". Government information quarterly, v. 34, n. 3, pp. 545-555.

https://doi.org/10.1016/j.giq.2017.05.002

Alonso, Sergio; Cabrerizo, Francisco-Javier; Herrera-Viedma, Enrique; Herrera, Francisco (2009). "h-Index: A review focused in its variants, computation and standardization for different scientific fields". Journal of informetrics, v. 3, n. 4, pp. $273-289$. https://doi.org/10.1016/j.joi.2009.04.001

Callon, Michael; Courtial, Jean-Pierre; Laville, Françoise (1991). "Co-word analysis as a tool for describing the network of interactions between basic and technological research: The case of polymer chemsitry". Scientometrics, v. 22, n. 1, pp. $155-205$. https://doi.org/10.1007/BF02019280

Cano, Virginia (1999). "Bibliometric overview of library and information science research in Spain". Journal of the American Society for Information Science, v. 50, n. 8, pp. 675-680.

https://doi.org/10.1002/(SICI)1097-4571(1999)50:8<675::AIDASI5>3.0.CO;2-B

Chavarro, Diego; Ràfols, Ismael; Tang, Puay (2018). "To what extent is inclusion in the Web of Science an indicator of journal 'quality'?". Research evaluation, v. 27, n. 2, pp. 106-118.

https://doi.org/10.1093/reseval/rvy001

Cobo, Manuel-Jesús (2012). SciMat: herramienta software para el análisis de la evolución del conocimiento científico. Propuesta de una metodología de evaluación. Tesis doctoral. Granada: Universidad de Granada.

Cobo, Manuel-Jesús; López-Herrera, Antonio-Gabriel; Herrera-Viedma, Enrique; Herrera, Francisco (2011). "Science mapping software tools: Review, analysis, and cooperative study among tools". Journal of the American Society for Information Science and Technology, v. 62, n. 7, pp. 1382-1402.

https://doi.org/10.1002/asi.21525

Cobo, Manuel-Jesús; López-Herrera, Antonio-Gabriel; Herrera-Viedma, Enrique; Herrera, Francisco (2012). "SciMAT: A new science mapping analysis software tool". Journal of the American Society for Information Science and Technology, v. 63, n. 8 , pp. $1609-1630$.

https://doi.org/10.1002/asi.22688

España (2019). "Resolución de 12 de noviembre de 2019, de la Comisión Nacional Evaluadora de la Actividad Investigadora, por la que se publican los criterios específicos aprobados para cada uno de los campos de evaluación". Boletín oficial del Estado, n. 284, 26 noviembre, pp. 130004-130024.

https://www.boe.es/eli/es/res/2019/11/12/(10)

Ferran-Ferrer, Núria; Guallar, Javier; Abadal, Ernest; Server, Adan (2017). "Research methods and techniques in Spanish library and information science journals (2012-2014)". Information research, v. 22, n. 1.

http://InformationR.net/ir/22-1/paper741.html 
Figuerola, Carlos G.; García-Marco, Francisco-Javier; Pinto, María (2017). "Mapping the evolution of library and information science (1978-2014) using topic modeling on LISA". Scientometrics, v. 112, n. 3.

https://doi.org/10.1007/s11192-017-2432-9

González-Alcaide, Gregorio; Alonso-Arroyo, Adolfo; Valderrama-Zurián, Juan-Carlos; Aleixandre-Benavent, Rafael (2008). "Una década de investigaciones en Anales de documentación (1998-2007): aproximación bibliométrica y temática". Anales de documentación, v. 11, pp. 57-78.

http://revistas.um.es/analesdoc/article/view/24811

Guallar, Javier; Ferran-Ferrer, Núria; Abadal, Ernest; Server, Adan (2017). "Revistas científicas españolas de información y documentación: análisis temático y metodológico". El profesional de la información, v. 26, n. 5, pp. 947-960.

https://doi.org/10.3145/epi.2017.sep.16

He, Qin (1999). “Knowledge discovery through co-word analysis”. Library trends, v. 48, n. 1, pp. 133-159.

http://hdl.handle.net/2142/8267

Herrera-Viedma, Enrique; López-Robles, José-Ricardo; Guallar, Javier; Cobo, Manuel-Jesús (2020). "Global trends in coronavirus research at the time of Covid-19: A general bibliometric approach and content analysis using SciMAT". EI profesional de la información, v. 29, n. 3.

https://doi.org/10.3145/epi.2020.may.22

Hirsch, Jorge E. (2005). "An index to quantify an individual's scientific research output". Proceedings of the National Academy of Sciences, v. 102, n. 46, pp. 16569-16572.

https://doi.org/10.1073/pnas.0507655102

Jiménez-Hidalgo, Sonia (2007). "Análisis de la autoría en la Revista española de documentación científica (1997-2005)". Revista española de documentación científica, v. 30, n. 3, pp. 305-322.

https://doi.org/10.3989/redc.2007.v30.i3.387

Kawalec, Anna (2013). "Research trends in library and information science based on Spanish scientific publication 2000 to 2010". Malaysian journal of library \& information science, v. 18, n. 2, pp. 1-13.

http://ejum.fsktm.um.edu.my/article/1370.pdf

López-Herrera, Antonio-Gabriel; Herrera-Viedma, Enrique; Cobo, Manuel-Jesús; Martínez, María-Ángeles; Kou, Gang; Shi, Yong (2012). "A conceptual snapshot of the first decade (2002-2011) of the International journal of information technology \& decision making". International journal of information technology \& decision making, v. 11, n. 2, pp. $247-270$. https://doi.org/10.1142/S0219622012400020

López-López, Pedro; Díaz-Hernández, Rosa-Ana; Angulo-Benítez, Sara; Giménez-Toledo, Elea (2001). “Estudio bibliométrico y de calidad de la Revista general de información y documentación (1991-2000)". Revista general de información y documentación, v. 11, n. 1, pp. 11-32.

http://hdl.handle.net/10261/11582

López-Robles, José-Ricardo; Guallar, Javier; Otegi-Olaso, José-Ramón; Gamboa-Rosales, Nadia-Karina (2019a). “El profesional de la información (EPI): bibliometric and thematic analysis (2006-2017)”. El profesional de la información, v. 28, n. 4, e280417.

https://doi.org/10.3145/epi.2019.jul.17

López-Robles, José-Ricardo; Guallar, Javier; Gamboa-Rosales, Nadia-Karina; Otegi-Olaso, José-Ramón; Cobo, Manuel-Jesús (2019b). "Mapa de la estructura intelectual de El profesional de la información de 2014 a 2018". Hipertext. net, n. 19, pp. 115-125.

https://doi.org/10.31009/hipertext.net.2019.i19.09

López-Robles, José-Ricardo; Otegi-Olaso, José-Ramón; Arcos, Rubén; Gamboa-Rosales, Nadia-Karina; Gamboa-Rosales, Hamurabi (2018). "Mapping the structure and evolution of JISIB: A bibliometric analysis of articles published in the Journal of intelligence studies in business between 2011 and 2017". Journal of intelligence studies in business, v. 8, n. 3. https://ojs.hh.se/index.php/JISIB/article/view/325

López-Robles, José-Ricardo; Otegi-Olaso, José-Ramón; Porto-Gómez, Igone; Cobo, Manuel-Jesús (2019c). "30 years of intelligence models in management and business: A bibliometric review". International journal of information management, v. 48, pp. 22-38.

https://doi.org/10.1016/j.ijinfomgt.2019.01.013

López-Robles, José-Ricardo; Otegi-Olaso, José-Ramón; Porto-Gómez, Igone; Gamboa-Rosales, Hamurabi; Gamboa-Rosales, Nadia-Karina (2020a). "La relación entre inteligencia de negocio e inteligencia competitiva: un análisis retrospectivo y bibliométrico de la literatura de 1959 a 2017". Revista española de documentación científica, v. 43, n. 1, e256.

https://doi.org/10.3989/redc.2020.1.1619 
López-Robles, José-Ricardo; Otegi-Olaso, José-Ramón; Porto-Gómez, Igone; Gamboa-Rosales, Hamurabi; Gamboa-Rosales, Nadia-Karina (2020b). "Understanding the intellectual structure and evolution of competitive intelligence: a bibliometric analysis from 1984 to 2017". Technology analysis \& strategic management, v. 32, n. 5, pp. 604-619.

https://doi.org/10.1080/09537325.2019.1686136

Martínez, María-Ángeles; Herrera, Manuel; López-Gijón, Javier; Herrera-Viedma, Enrique (2014). “H-Classics: characterizing the concept of citation classics through H-index". Scientometrics, v. 98, n. 3, pp. 1971-1983.

https://doi.org/10.1007/s11192-013-1155-9

Mongeon, Philippe; Paul-Hus, Adèle (2016). "The journal coverage of Web of Science and Scopus: A comparative analysis". Scientometrics, v. 106, n. 1, pp. 213-228.

https://doi.org/10.1007/s11192-015-1765-5

Moral-Muñoz, José-Antonio; Cobo, Manuel-Jesús; Chiclana, Francisco; Collop, Andrew; Herrera-Viedma, Enrique (2016). "Analyzing highly cited papers in intelligent transportation systems". IEEE transactions on intelligent transportation systems, v. 17, n. 4, pp. 993-1001.

https://doi.org/10.1109/TITS.2015.2494533

Moral-Muñoz, José-Antonio; Herrera-Viedma, Enrique; Santisteban-Espejo, Antonio; Cobo, Manuel-Jesús (2020). "Software tools for conducting bibliometric analysis in science: an up-to-date review". El profesional de la información, v. 29, n. 1, e290103.

https://doi.org/10.3145/epi.2020.ene.03

Nederhof, Anton J. (2006). "Bibliometrics monitoring of research performance in the social sciences and humanities: A review". Scientometrics, v. 66, n. 1, pp. 81-100.

https://doi.org/10.1007/s11192-006-0007-2

Olmeda-Gómez, Carlos; Ovalle-Perandones, María-Antonia; Perianes-Rodríguez, Antonio (2017). "Co-word analysis and thematic landscapes in Spanish information science literature, 1985-2014". Scientometrics, v. 113, n. 1, pp. $195-217$. https://doi.org/10.1007/s11192-017-2486-8

Ollé-Castellà, Candela; Porras, Mercè (2008). “Una mirada als vint números de BiD”. BiD: textos universitaris de biblioteconomia i documentació, n. 21.

https://doi.org/10.1344/105.000000330

Pérez-Álvarez-Ossorio, José-Ramón (1997). “Cobertura temática y procedencia institucional de los artículos publicados en la Revista española de documentación científica en sus veinte años de existencia". Revista española de documentación científica, v. 20, n. 3, pp. 290-298.

https://doi.org/10.3989/redc.1997.v20.i3.584

Pérez-Cabezas, Verónica; Ruiz-Molinero, Carmen; Carmona-Barrientos, Inés; Herrera-Viedma, Enrique; Cobo, Manuel-Jesús; Moral-Muñoz, José-Antonio (2018). "Highly cited papers in Rheumatology: identification and conceptual analysis". Scientometrics, v. 116, n. 1, pp. 555-568.

https://doi.org/10.1007/s11192-018-2712-z

Powell, Taman H.; Kouropalatis, Yiannis; Morgan, Robert E.; Karhu, Päivi (2016). “Mapping knowledge and innovation research themes: Using bibliometrics for classification, evolution, proliferation and determinism". International journal of entrepreneurship and innovation management, v. 20, n. 3-4, pp. 174-199.

https://doi.org/10.1504/IJEIM.2016.077960

Sánchez-Gil, Susana; Gorraiz, Juan; Melero-Fuentes, David (2018). "Reference density trends in the major disciplines". Journal of informetrics, v. 12, n. 1, pp. 42-58. https://doi.org/10.1016/j.joi.2017.11.003.

Sanz-Casado, Elías; Lascurain, María-Luisa; Iribarren, Isabel (2007). "Luces y sombras en la evaluación de la investigación en Ciencias Sociales y Humanidades". En: La evaluación de la actividad científica en ciencias sociales y humanidades. Bilbao: Universidad del País Vasco, pp. 15-32.

https://web-argitalpena.adm.ehu.es/pdf/UHPDF079105.pdf

Schreiber, Michael (2007). "Self-citation corrections for the Hirsch index". Europhysics letters (EPL), v. 78, n. 3, 30002. https://doi.org/10.1209/0295-5075/78/30002

Schubert, András (2007). "Successive h-indices". Scientometrics, v. 70, n. 1, pp. 201-205. https://doi.org/10.1007/s11192-007-0112-x

Sedighi, Mehri (2016). "Application of word co-occurrence analysis method in mapping of the scientific fields (case study: the field of Informetrics)". Library review, v. 65, n. 1/2, pp. 52-64.

https://doi.org/10.1108/LR-07-2015-0075 
Somoza, Marta; Guallar, Javier; Rodríguez-Gairín, Josep-Manuel; Abadal, Ernest (2017). “Presencia de revistas españolas en bases de datos internacionales". En: Abadal, Ernest (ed.). Revistas científicas: situación actual y retos de futuro. Barcelona: Universitat de Barcelona, pp. 161-178. ISBN: 9788491680383

http://eprints.rclis.org/32136

Sternitzke, Christian; Bergmann, Isumo (2009). "Similarity measures for document mapping: A comparative study on the level of an individual scientist". Scientometrics, v. 78, n. 1, pp. 113-130.

https://doi.org/10.1007/s11192-007-1961-z

Wang, Jian (2013). "Citation time window choice for research impact evaluation”. Scientometrics, v. 94, n. 3, pp. $851-872$. https://doi.org/10.1007/s11192-012-0775-9

Wang, Wanru; Laengle, Sigifredo; Merigó, José M.; Yu, Dejian; Herrera-Viedma, Enrique; Cobo, Manuel-Jesús; Bouchon-Meunier, Bernadette (2018). "A bibliometric analysis of the first twenty-five years of the International journal of uncertainty, fuzziness and knowledge-based systems". International journal of uncertainty, fuzziness and knowledge-based systems, v. 26, n. 02, pp. 169-193.

https://doi.org/10.1142/S0218488518500095

\section{Anexos}

\section{A.1. Análisis anual de principales temas de investigación 2015-2019}

Para visualizar y analizar la evolución de las revistas españolas de Information Science \& Library Science en WoS de 2015 a 2019, se han confeccionado los diagramas estratégicos de cada año, así como el mapa de evolución para el área al completo.

Tomando en cuenta los diagramas estratégicos, en la tabla A.1 se recogen los principales temas de investigación desarrollados y su rendimiento según el número de publicaciones de 2015 a 2019.

Tabla A.1. Principales temas de investigación 2015-2019

\begin{tabular}{|c|c|c|c|c|c|c|}
\hline \multirow[b]{2}{*}{ Temas } & \multirow{2}{*}{$\begin{array}{c}\text { Publicaciones } \\
\text { (citas | h-index } \\
\text { máx.) }\end{array}$} & \multicolumn{5}{|c|}{ Evolución (cuadrante) } \\
\hline & & 2015 & 2016 & 2017 & 2018 & 2019 \\
\hline ACADEMIC-LIBRARIES & $124(177 \mid 6)$ & $\mathrm{C} 1$ & $\mathrm{C} 4$ & $\mathrm{C} 1$ & & $\mathrm{C} 1$ \\
\hline TWITTER & $71(185 \mid 7)$ & & $\mathrm{C} 1$ & & $\mathrm{C} 1$ & \\
\hline SOCIAL-NETWORKS & $64(386 \mid 9)$ & $\mathrm{C} 1$ & & $\mathrm{C} 4$ & & \\
\hline BIBLIOMETRIC-INDICATORS & $55(205$ | 6) & $\mathrm{C} 1$ & & & $\mathrm{C} 1$ & \\
\hline SOCIAL-MEDIA & $51(13 \mid 2)$ & & & & & $\mathrm{C} 1$ \\
\hline DIGITAL-MEDIA & $41(29 \mid 2)$ & & & & $\mathrm{C} 3$ & $\mathrm{C} 1$ \\
\hline BIG-DATA & $40(110 \mid 5)$ & & $\mathrm{C} 1$ & & $\mathrm{C} 4$ & \\
\hline ELECTORAL-CAMPAIGNS & $38(151 \mid 6)$ & & & $\mathrm{C} 1$ & & \\
\hline SCIENTIFIC-JOURNALS & $38(14 \mid 2)$ & & & & $\mathrm{C} 1$ & C4 \\
\hline PUBLIC-LIBRARIES & $35(34$ | 3$)$ & & & & $\mathrm{C} 4$ & \\
\hline CONTENT-ANALYSIS & $33(19 \mid 3)$ & & & & $\mathrm{C} 4$ & $\mathrm{C} 2$ \\
\hline OPEN-ACCESS & $32(64 \mid 4)$ & $\mathrm{C} 3$ & & $\mathrm{C} 1$ & & \\
\hline PROFESSIONAL-PROFILES & $31(78 \mid 5)$ & & $\mathrm{C} 1$ & & & \\
\hline SCIENTIFIC-PRODUCTIVITY & $31(40 \mid 3)$ & & & $\mathrm{C} 4$ & $\mathrm{C} 1$ & \\
\hline NEWS-MEDIA & $29(140 \mid 7)$ & $\mathrm{C} 1$ & & & & \\
\hline OPEN-DATA & $27(48 \mid 4)$ & $\mathrm{C} 3$ & & & & $\mathrm{C} 1$ \\
\hline INFORMATION-DESIGN & $25(50 \mid 4)$ & & & $\mathrm{C} 1$ & & \\
\hline KNOWLEDGE-MANAGEMENT & $25(21 \mid 3)$ & & $\mathrm{C} 2$ & & & \\
\hline PUBLIC-RELATIONS & $25(3 \mid 1)$ & & & & & $\mathrm{C} 1$ \\
\hline WEB-2.0 & $25(50 \mid 4)$ & & C4 & & & \\
\hline SCIENTIFIC-COMMUNICATION & $23(8 \mid 2)$ & & & & & $\mathrm{C} 1$ \\
\hline PUBLIC-INFORMATION & $21(75 \mid 5)$ & & & $\mathrm{C} 1$ & & \\
\hline FAKE-NEWS & $17(36 \mid 4)$ & & & & $\mathrm{C} 1$ & \\
\hline ALTERNATIVE-METRICS & $16(72 \mid 5)$ & & $\mathrm{C} 2$ & & & \\
\hline OPEN-SCIENCE & $15(5 \mid 1)$ & & & & & C4 \\
\hline KNOWLEDGE-ORGANIZATION & $13(11 \mid 2)$ & $\mathrm{C} 1$ & & & & \\
\hline DIGITAL-PRESERVATION & $10(1 \mid 1)$ & & & & & C3 \\
\hline DOCUMENT-MANAGEMENT & $10(10 \mid 2)$ & & $\mathrm{C} 3$ & & & \\
\hline
\end{tabular}




\begin{tabular}{|c|c|c|c|c|c|c|}
\hline COMMUNICATION-ETHICS & $9(2 \mid 1)$ & & & $\mathrm{C} 3$ & & \\
\hline CONTENT-CURATION & $9(17 \mid 3)$ & & $\mathrm{C} 3$ & & & \\
\hline INFORMATION-MANAGEMENT & $9(9 \mid 2)$ & $\mathrm{C} 3$ & & & & \\
\hline INFORMATION-SOURCES & $9(1 \mid 1)$ & & & & & $\mathrm{C} 3$ \\
\hline BIBLIOGRAPHIC-REFERENCES & $8(1 \mid 1)$ & & & & & C3 \\
\hline DIGITAL-LIBRARIES & $8(1 \mid 1)$ & & & & & $\mathrm{C} 2$ \\
\hline MACHINE-LEARNING & $8(17 \mid 3)$ & & & $\mathrm{C} 3$ & & \\
\hline COMMUNITY-MANAGERS & $7(15 \mid 2)$ & & & & $\mathrm{C} 2$ & \\
\hline FACT-CHECKING & $7(3 \mid 1)$ & & & & & $\mathrm{C} 3$ \\
\hline AUDIOVISUAL-CONTENT & $6(10 \mid 2)$ & & & $\mathrm{C} 2$ & & \\
\hline E-ADMINISTRATION & $6(7 \mid 2)$ & & & $\mathrm{C} 3$ & & \\
\hline INSTAGRAM & $6(10 \mid 2)$ & & & & $\mathrm{C} 3$ & \\
\hline PUBLIC-COMMUNICATION & $6(29 \mid 3)$ & & & $\mathrm{C} 2$ & & \\
\hline SOCIAL-NETWORKS-ANALYSIS & $6(1 \mid 1)$ & & & & & C3 \\
\hline MOBILE-DEVICES & $5(1 \mid 1)$ & & & & & $\mathrm{C} 2$ \\
\hline USER-GENERATED-CONTENT & $5(4 \mid 2)$ & & & & & C4 \\
\hline VIDEOGAMES & $5(1 \mid 1)$ & & & & $\mathrm{C} 3$ & \\
\hline INFORMATION-POLICIES & $4(1 \mid 1)$ & & & & $\mathrm{C} 3$ & \\
\hline LINKED-DATA & $4(1 \mid 1)$ & & & & $\mathrm{C} 2$ & \\
\hline NETFLIX & $3(7 \mid 2)$ & & & & $\mathrm{C} 2$ & \\
\hline
\end{tabular}

En el gráfico A.1 se muestra el diagrama estratégico para la anualidad 2015. En este año se identificaron 8 temas repartidos en los cuadrantes de temas motor y temas emergentes o en declive, siendo los primeros los que tienen una mayor presencia en el mapa estratégico.

En 2015, de los 8 temas identificados, tan solo ACADEMIC-LIBRARIES, SOCIAL-NETWORKS, NEWS-MEDIA, BIBLIOMETRIC INDICATORS y KNOWLEDGE-ORGANIZATION pueden ser considerados relevantes para el desarrollo y consolidación del área de conocimiento, siendo sus principales componentes los siguientes:

- SOCIAL-NETWORKS: Hypermedia, Facebook, Alternative-Metrics, Web 2.0, Twitter, Tumblr, Transmedia, Social Media, Public Libraries e Internet Use.

- ACADEMIC-LIBRARIES: Information Literacy, Embedded Librarians, e-Books, Social Media Marketing, Scientific Output, Scientific Communication, School Libraries, Open Software, Library Services, Integrated Library Systems e Information Systems.

- NEWS-MEDIA: Cyberjournalism, Credibility, Blogs, User Studies, Online Media, Online Journalism, Mass Media, Freedom of Expression, Digital Storytelling, Digital Journalism y Cybermedia.

- KNOWLEDGE-ORGANIZATION: Content Analysis, Archival Science, Library Science, Knowledge Representation y Knowledge Organization Systems.

- BIBLIOMETRIC INDICATORS: H-index, Citation Analysis, Bibliometric Analysis, University Presses, Smartphones, Scientific Journals, Research Evaluation, Mobile Applications e Impact Factor.

En el gráfico A.2 se muestra el diagrama estratégico de 2016, donde se han identificado 9 temas repartidos en los cuatro cuadrantes, siendo los de temas motor y básicos o transversales los que concentran más publicaciones y citas.

De 2015 a 2016 se ha registrado un aumento sencillo en el número de temas, así como un reparto en los cuadrantes, propiciado principalmente por la disponibilidad de un período anterior y sobre el cual se han producido modificaciones en la estructura intelectual del área de conocimiento.

En 2016, de los 9 temas identificados, TWITTER, PROFESSIONAL-PROFILES, BIG-DATA, WEB-2.0 y ACADEMIC-LIBRARIES pueden ser considerados relevantes para el desarrollo y consolidación del área de conocimiento, siendo sus principales componentes los siguientes:

- TWITTER: Social Media, Social Audience, Metamedia, Focus Groups, Facebook, Active Audiences, YouTube, Traditional Media, Social TV, Social Networks y Social Networking Sites.

- PROFESSIONAL-PROFILES: Information Professionals, Digital Journalism, Data Journalism, Cybermedia, Public Libraries, Online Media, Online Journalism, Library Services, Librarianship, Journalism e Information Skills.

- WEB-2.0: Digital Communication, Digital Book, Citizen Participation, Transmedia, Social Web, Scientific Communication, Public Relations, Political Parties, Mobile Devices, Knowledge Society e Information Society. 
- ACADEMIC-LIBRARIES: Data Envelopment Analysis, Content Analysis, Open Access, Information Literacy, Geographic Information Systems, e-Learning y e-Books.

- BIG-DATA: Information Dissemination, Data Mining, Business Intelligence, Smart Cities, Sentiment Analysis, Open Government, Open Data, Machine Learning e Information Retrieval.

Es importante resaltar que ACADEMIC-LIBRARIES es el único tema que repite entre los más relevantes con respecto al período anterior, pasando de ser motor a básico o transversal. En términos de estructura, los temas guardan relación, manteniéndose algunos en el tiempo y renovando otros.

En el gráfico A.3 se muestra el diagrama estratégico de 2017, donde se han identificado 12 temas repartidos en los cuatro cuadrantes, siendo los correspondientes a motor y básicos o transversales los que concentran más publicaciones y citas.

De 2016 a 2017 se ha registrado un aumento importante en el número de temas, manteniendo un equilibrio en el reparto de estos dentro de los cuadrantes.

En 2017, de los 12 temas identificados, ELECTORAL-CAMPAIGNS, INFORMATION-DESIGN, PUBLIC INFORMATION, OPEN ACCESS, ACADEMIC LIBRARIES, SOCIAL NETWORKS y SCIENTIFIC PRODUCTIVITY pueden ser considerados relevantes para el desarrollo y consolidación del área de conocimiento, siendo sus principales componentes:

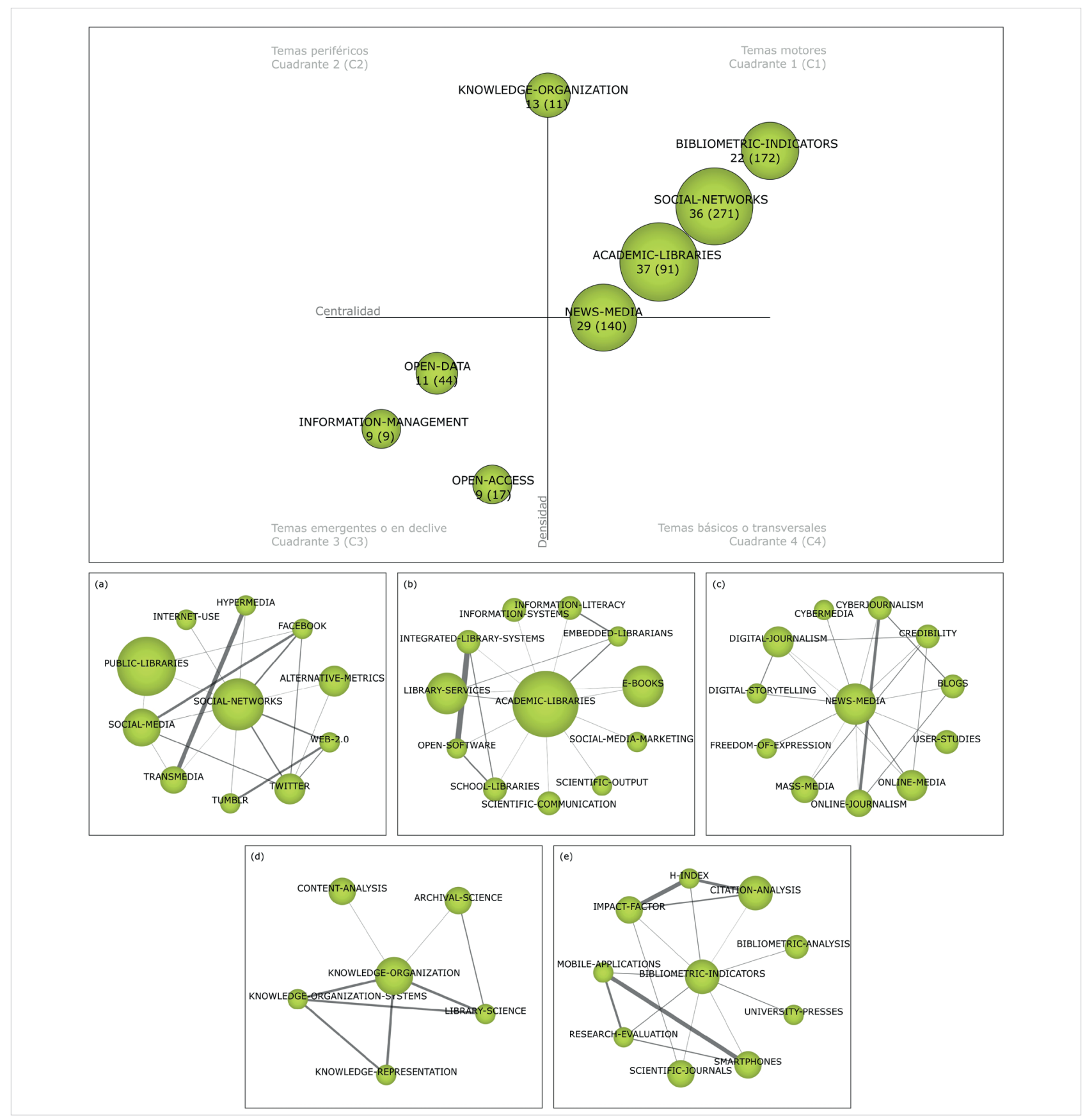

Gráfico A.1 Diagramas estratégicos 2015 y redes tematicas de los temas motores (C1) y temas básicos y transversales (C4) 
- EleCtORAL-CAMPAigNS: Political Communication, Mediatization, Media Coverage, Infotainment, Twitter, Social Networks Analysis, Social Networking Sites, Social Media, Political Parties, Political Marketing y Political Information.

- SOCIAL NETWORKS: Digital Communication, Cultural Heritage, Active Citizenship, Television Fiction, Scripted Television, Local Elections, Internet Use, Instagram, General Election, Facebook y Digital Empowerment.

- INFORMATION-DESIGN: Data Visualization, Cybermedia, Bibliographic Review, Web Design, Transmedia, Printed Press, Online Journalism, News Media, Journalism, Information Visualization y Digital Press.

- PUBLIC INFORMATION: e-Government, Communication Management, City Councils, Web 2.0, Public Management, Press Rooms, Open Data, Local Governments, Information Reuse, Information Dissemination y Governance.

- OPEN ACCESS: Data Mining, Data Analysis, Alternative Metrics, User Studies, Science 2.0, Scholarly Communication, Institutional Repositories, Information Retrieval, Impact Factor, Human Computer Interaction y Digital Libraries.

- ACADEMIC LIBRARIES: Library Services, Information Management, Information Literacy, Web Portals, Verbal Protocol, Subject Cataloguing, Smartphones, Plagiarism, Online Education y Lifelong Learning.

- SCIENTIFIC PRODUCTIVITY: Co-Authorship Networks, Bibliographic Databases, Scientific Journals, Scientific Communications, Knowledge Organization, Editorial Committee y Digitization.

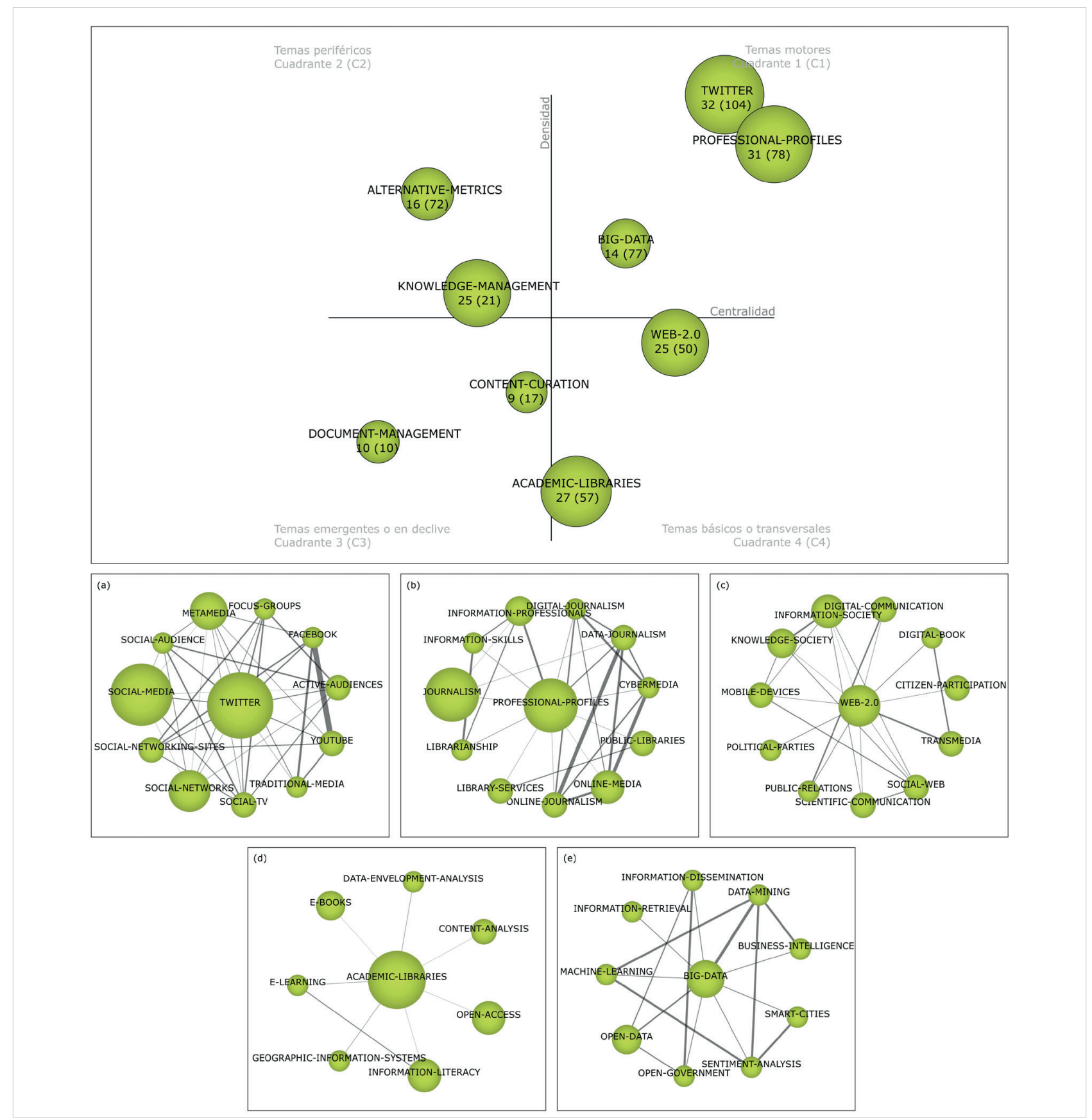

Gráfico A.2. Diagramas estratégicos 2016 y redes tematicas de los temas motores (C1) y temas básicos y transversales (C4) 
A igual que el período anterior, ACADEMIC-LIBRARIES repite como un tema relevante para el desarrollo del área de conocimiento y cambia nuevamente de cuadrante, ubicándose como motor. Estos cambios vienen dados por la renovación de las estructuras interiores y los temas cubiertos por este.

En el gráfico A.4 se muestra el diagrama estratégico de 2018, donde se han identificado 15 temas repartidos en los cuatro cuadrantes, siendo los correspondientes a los temas motor y básicos o transversales los que concentran más publicaciones y citas, respectivamente.

Al igual que en el período anterior, de 2017 a 2018 se ha registrado un aumento importante en el número de temas, manteniendo un equilibrio en el reparto de estos dentro de los cuadrantes.

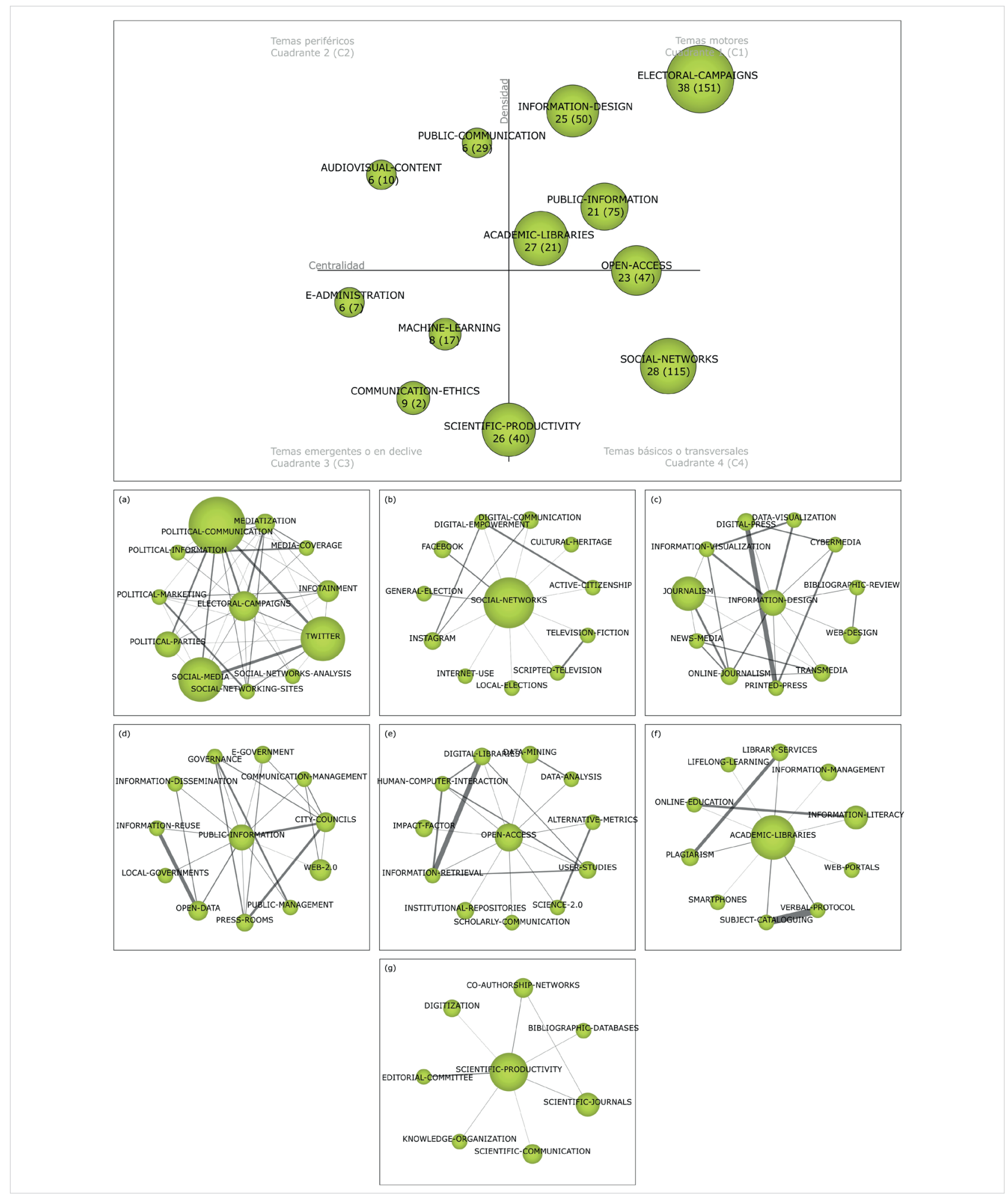

Gráfico A.3. Diagramas estratégicos 2017 y redes tematicas de los temas motores (C1) y temas básicos y transversales (C4) 
En 2018, de los 15 temas identificados, TWITTER, FAKE-NEWS, SCIENTIFIC-JOURNALS, BIBLIOMETRIC-INDICATORS, SCIENTIFIC-PRODUCTIVITY, BIG-DATA, CONTENT-ANALYSIS y PUBLIC-LIBRARIES pueden ser considerados relevantes para el desarrollo y consolidación del área de conocimiento, siendo sus principales componentes los siguientes:

- BIBLIOMETRIC-INDICATORS: Normalized Citation, Bibliographical Production, Alternative Metrics, Wikipedia, Spanish Universities, Scientific Production, Scientific Evaluation, Research Policy, Research Performance, Research Evaluation y Open Access.

- TWITTER: Influencers, Facebook, Dialogic Communication, Agenda Setting, Social Networks, Social Media, Populism, Political Information, Political Communication, Personal Branding y Journalism.
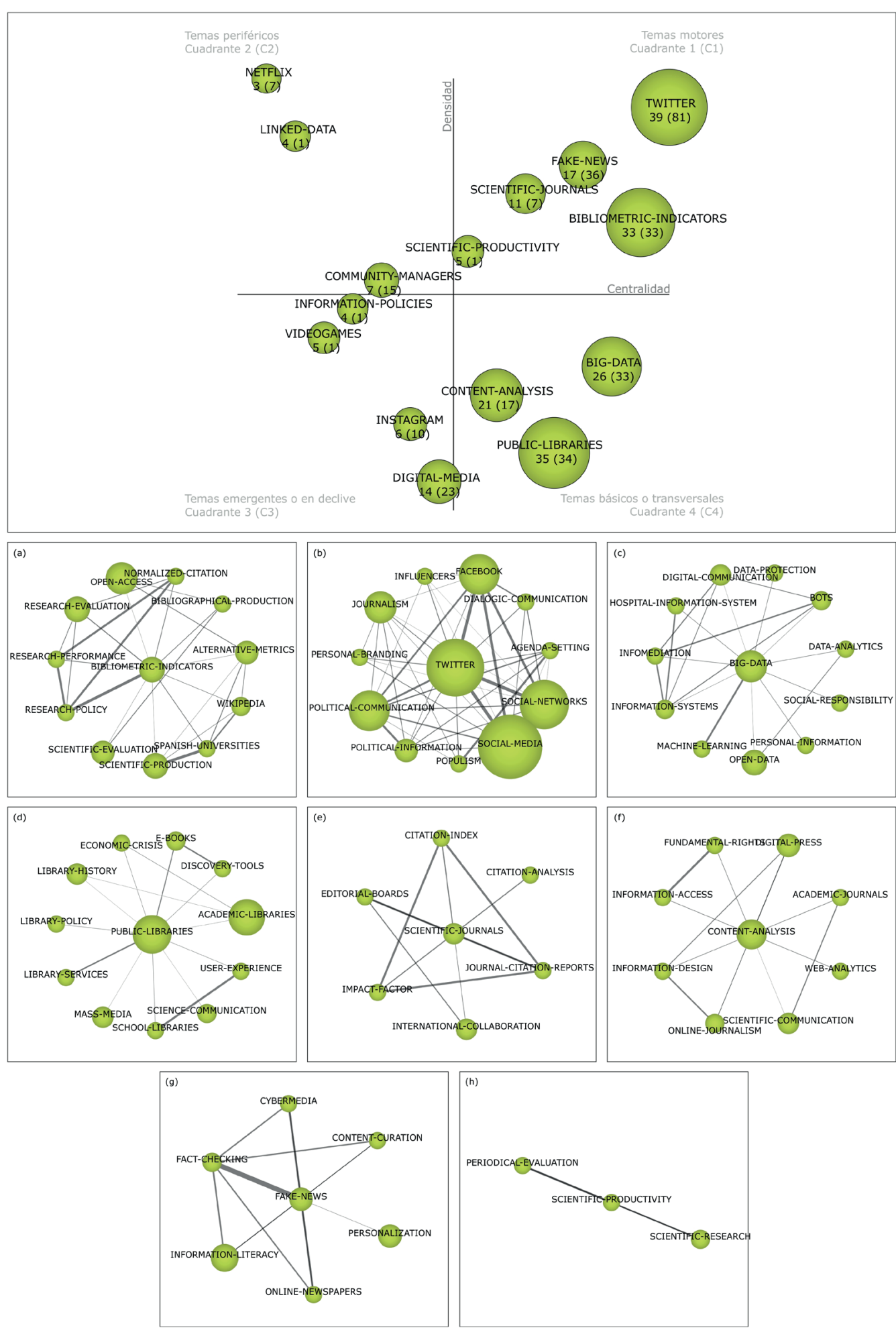

Gráfico A.4. Diagramas estratégicos 2018 y redes tematicas de los temas motores (C1) y temas básicos y transversales (C4) 
- BIG-DATA: Data Protection, Bots, Data Analytics, Social Responsibility, Personal Information, Open Data, Machine Learning, Information Systems, Infomediation, Hospital Information Systems y Digital Communication.

- PUBLIC-LIBRARIES: e-Books, Discovery Tools, Academic Libraries, User Experience, Science Communication, School Libraries, Mass Media, Library Services, Library Policy, Library History y Economic Crisis.

- SCIENTIFIC-JOURNALS: Citation Index, Citation Analysis, Journal Citation Reports, International Collaboration, Impact Factor y Editorial Boards.

- CONTENT-ANALYSIS: Digital Press, Academic Journals, Web Analytics, Scientific Communication, Online Journalism, Information Design, Information Access y Fundamental Rights.
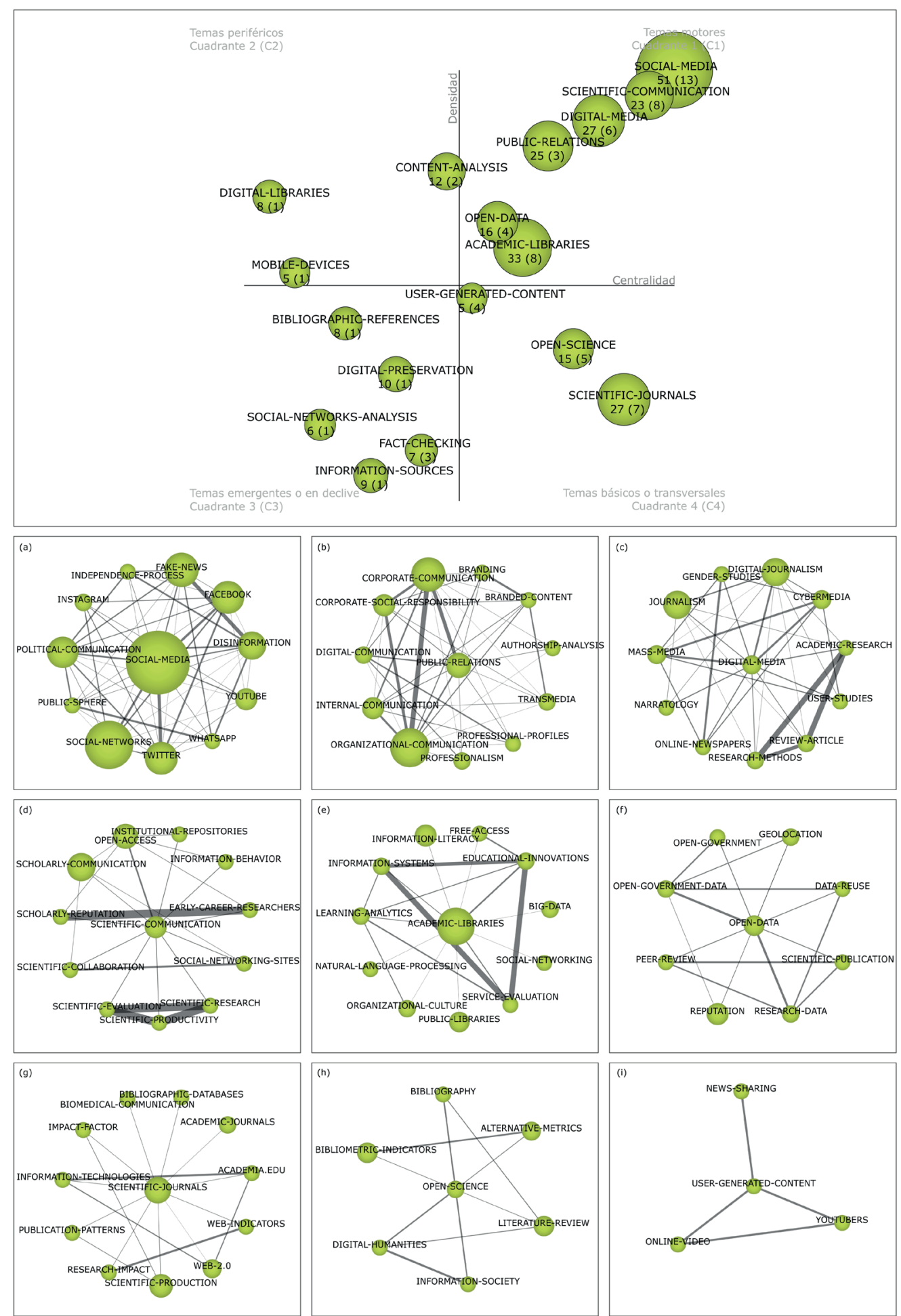

Gráfico A.5. Diagramas estratégicos 2019 y redes tematicas de los temas motores (C1) y temas básicos y transversales (C4) 
- FAKE-NEWS: Cybermedia, Content Curation, Personalization, Online Newspapers, Information Literacy y Fact Checking.

- SCIENTIFIC-PRODUCTIVITY: Scientific Research y Periodical Evaluation.

En este período, solo repite SCIENTIFIC-PRODUCTIVITY, que pasa de ser básico o transversal a motor. Se observa que temas que aparecían en períodos anteriores reaparecen dentro del mapa estratégico dando continuidad a su evolución.

Por último, en el gráfico A.5 se muestra el diagrama estratégico de 2019, donde se han identificado 17 temas repartidos en los cuatro cuadrantes, siendo los correspondientes a los temas motor, emergentes o en declive y básicos o transversales los que concentran más publicaciones y citas.

De 2018 a 2019 se ha registrado un aumento importante en el número de temas, manteniendo un equilibrio en el reparto de estos dentro de los cuadrantes.

En 2019, de los 17 temas identificados, SOCIAL-MEDIA, SCIENTIFIC-COMMUNICATION, DIGITAL-MEDIA, PUBLIC-RELATIONS, OPEN-DATA, ACADEMIC-LIBRARIES, USER-GENERATED-CONTENT, OPEN-SCIENCE y SCIENTIFIC-JOURNALS PUEden ser considerados relevantes para el desarrollo y consolidación del área de conocimiento, siendo sus principales componentes los siguientes:

- SOCIAL-MEDIA: Fake News, Facebook, Disinformation, YouTube, WhatsApp, Twitter, Social Networks, Public Sphere, Political Communication, Instagram e Independence Process.

- PUBLIC-RELATIONS: Branding, Branded Content, Authorship Analysis, Transmedia, Professional Profiles, Professionalism, Organizational Communication, Internal Communication, Digital Communication, Corporate Social Responsibility y Corporate Communication.

- DIGITAL-MEDIA: Digital Journalism, Cybermedia, Academic Research, User Studies, Review Article, Research Methods, Online Newspapers, Narratology, Mass Media, Journalism y Gender Studies.

- SCIENTIFIC-COMMUNICATION: Institutional Repositories, Information Behavior, Early Career Researchers, Social Networking Sites, Scientific Research, Scientific Productivity, Scientific Evaluation, Scientific Collaboration, Scholarly Reputation, Scholarly Communication y Open Access.

- OPEN-DATA: Geolocation, Data Reuse, Scientific Publication, Research Data, Reputation, Peer Review, Open Government Data y Open Government.

- ACADEMIC-LIBRARIES: Educational Innovations, Big Data, Social Networking, Service Evaluation, Public Libraries, Organizational Culture, Natural Language Processing, Learning Analytics, Information Systems e Information Literacy.

- OPEN-SCIENCE: Bibliography, Alternative Metrics, Literature Review, Information Society, Digital Humanities y Bibliometric Indicators.

- SCIENTIFIC-JOURNALS: Bibliographic Databases, Academic Journals, Academia.edu, Web Indicators, Web 2.0, Research Impact, Publication Patterns, Information Technologies, Impact Factor y Biomedical Communication.

- USER-GENERATED-CONTENT: Youtubers, Online Video y News Sharing.

En este período, SCIENTIFIC-JOURNALS repite como uno de los temas nucleares para el campo de conocimiento, y pasa de ser motor a básico o transversal.

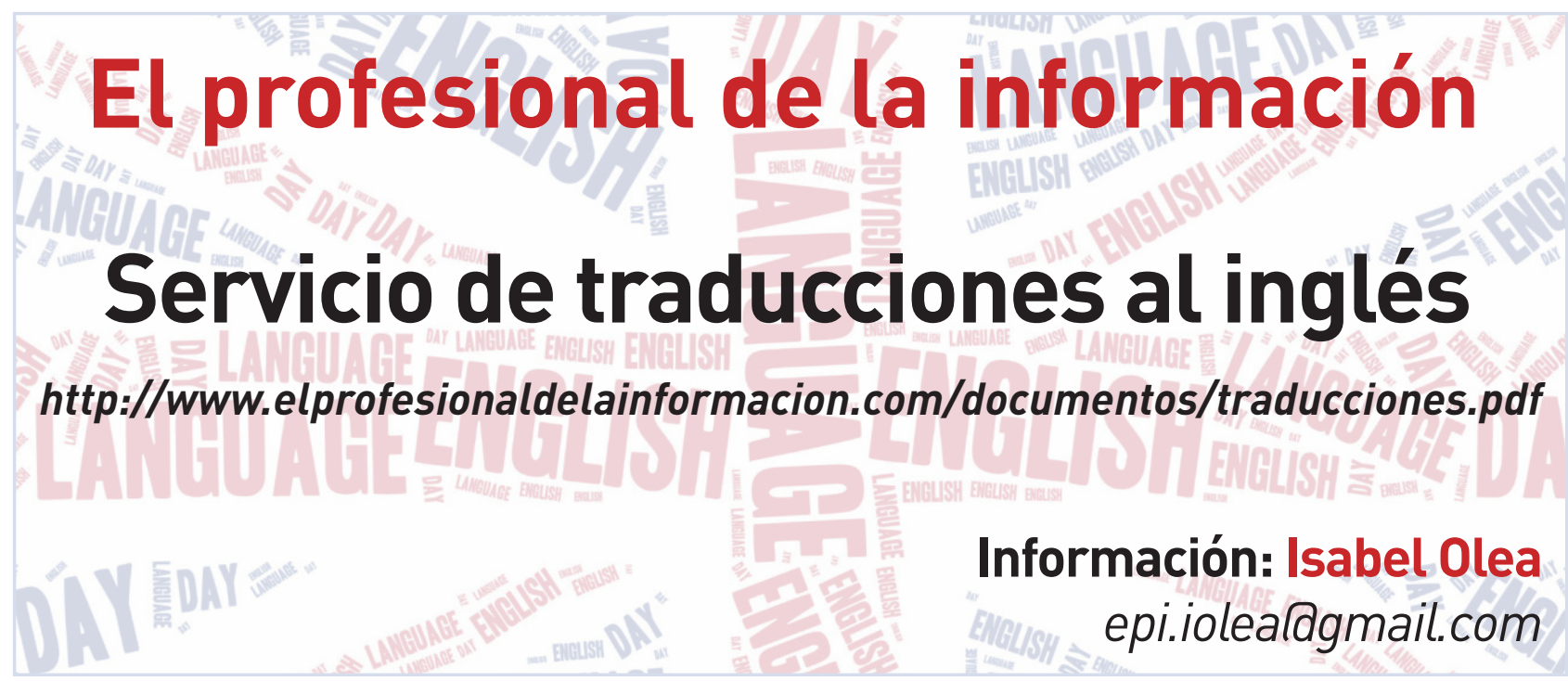

